\title{
Principaux critères économiques de gestion des forêts : analyse critique et comparative
}

\author{
Jean-Luc Peyron ${ }^{\mathrm{a} *}$, Jean-Philippe Terreaux ${ }^{\mathrm{b}}$, \\ Philippe Calvet ${ }^{\mathrm{c}}$, Bin Guo ${ }^{\mathrm{d}}$
}

\author{
${ }^{a}$ Engref/Inra, 14, rue Girardet, CS 4216, 54042 Nancy cedex, France \\ bemagref, groupement de Montpellier, domaine de Lavalette, \\ 361, rue Jean-François-Breton, BP 5095, 34033 Montpellier cedex 1, France \\ 'Inra, Station de recherches forestières de Pierroton, domaine de l'Hermitage, \\ BP 45, 33611 Gazinet cedex, France \\ ${ }^{\mathrm{d}} 7$, rue Antoine Bourdelle, 75015 Paris, France
}

(Reçu le 12 mars 1996 ; révisé le 25 juin 1996 ; accepté le 30 septembre 1997)

Résumé - Les principaux critères utilisés dans le cadre de la gestion des peuplements forestiers sont présentés et commentés : leur domaine de validité est circonscrit, la terminologie correspondante est précisée, les aspects historiques sont mentionnés. L'analyse réalisée ne prend pas en compte les biens et services non marchands et se place délibérément dans un contexte certain et sans risques. Mais elle considère aussi bien les structures de peuplement équiennes qu'inéquiennes, la gestion actuelle que le long terme, les formulations complètes que simplifiées. En outre, même si le marché financier est supposé parfait dans la majorité des développements, les conséquences de son imperfection sont examinées.

Le tableau I présente une structuration des différents critères de gestion et sert de référence à la fois pour un peuplement équienne considéré à long terme, lors d'une recherche de rendements soutenus et pour un peuplement quelconque en cours de croissance. Cette structuration repose d'une part sur la nature générale des critères, d'autre part sur la variable considérée. On distingue ainsi les critères d'accumulation qui caractérisent un capital, les critères de productivité qui s'intéressent au produit annuel de ce capital, les critères de rentabilité qui relativisent ce produit par rapport à des investissements concurrents. Quant aux variables, elles consistent soit en une quantité (volume), soit en un revenu, soit encore en un bénéfice considéré sur une période limitée ou illimitée.

Les conséquences majeures, sur la gestion forestière optimale, de l'un ou l'autre de ces critères sont décrites dans le cas d'un peuplement équienne. Les figures 1 à 4 illustrent, dans un cas simplifié et le plan (âge, revenu), les différences auxquelles ces derniers conduisent lors de la recherche du meilleur âge d'exploitabilité. La figure 5 fait de même dans le plan (âge, taux d’accroissement du revenu). Deux exemples complets sont présentés qui portent sur le pin mari-

* Correspondance et tirés à part

Courriel : peyron@engref.fr 
time dans les Landes de Gascogne pour l'un, l'épicéa commun dans le nord-est de la France pour l'autre. Le tableau $I I$ récapitule les principales données de ces exemples tandis que les tableaux $I I I$ et $I V$ d'une part, les figures 6 et $7 \mathrm{~d}$ 'autre part, présentent les résultats obtenus pour trois critères : la production moyenne annuelle en volume, le bénéfice moyen annuel et le bénéfice actualisé sur une infinité de révolutions identiques. En particulier, la sylviculture optimale à long terme résultant de ce dernier critère apparaît comme étant relativement tempérée par rapport à celles correspondant aux deux premiers.

Dans l'approche théorique, le marché financier est généralement considéré comme parfait alors que, dans la réalité, il est foncièrement imparfait. La figure 8 illustre la différence de situation du consommateur dans ces deux cas. Elle incite ensuite à conclure que la théorie s'adapte sans difficultés à la réalité à condition d'utiliser comme taux d'actualisation le taux d'emprunt ou d'épargne suivant la situation d'emprunteur ou d'épargnant du propriétaire forestier considéré. (ㅇ Inra/Elsevier, Paris)

\title{
aménagement forestier / économie forestière / révolution / exploitabilité / actualisation
}

\begin{abstract}
Main economic management criteria for forests: a review. The main criteria used in the frame of forest stand management are presented and commented: their validity range is defined, the corresponding terminology is specified, historic facts are mentioned. The analysis does not take into account non-market goods and services; it is resolutely placed in an environment without uncertainty nor risks. However, it considers both even and uneven-aged forest structures, present management and long term, complete and simplified formulations. Moreover, although the financial market is supposed perfect in most circumstances, consequences of its imperfection are discussed.

Table I provides a structure of these different management criteria and constitutes a reference either for an even-aged stand in the long term, or with the objective of a sustained yield or for a growing stand. Such a structure depends on the one hand on the general nature of criteria, and on the variable under consideration on the other hand. A distinction can be made between accumulation criteria that characterise a capital, productivity criteria that concern the annual output of this capital, efficiency criteria that compare this output to other investment possibilities. As for variables, they are either a quantity (volume), or a revenue, or even a profit resulting from a finite or infinite period.

The major consequences of these criteria on optimal forest management are described in the case of an even-aged stand. Figures $I-4$ show, in a simplified case and for the output as a function of age, differences obtained from these criteria in the determination of the best rotation age. Figure 5 shows the same kind of result for the output rate of growth as a function of age. Two examples are presented for maritime pine in the south west of France, and for Norway spruce in the north east of France. Table II synthesises the main data used in these examples; tables III and $I V$ on the one hand and figures 6 and 7 on the other provide results obtained for three criteria: the mean annual increment in volume, the mean annual net revenue and the present net value of an infinite series of like rotations. For example, the optimal management in the long run associated with this last criterion appears relatively temperate in comparison to those corresponding to the first two ones.

When it is theoretically studied, the financial market is generally taken as perfect whereas, in reality, it is surely imperfect. Figure 8 shows the difference between these two cases as regards the consumer situation. One conclusion is that the theory can be adapted easily to the real world by using the rate used to borrow or lend money as discount rate according to the situation of borrower or lender of the forest owner under consideration. (C) Inra/Elsevier, Paris)
\end{abstract}

\section{forest planning / forest economics / rotation, maturity / discounting}




\section{INTRODUCTION}

L'optimisation de la gestion forestière a souvent consisté, pour des peuplements forestiers équiennes ${ }^{1}$, à se restreindre à la détermination de la révolution optimale ou, de manière équivalente, de l'âge optimal d'exploitation, ou encore de l'âge d'exploitabilité ${ }^{2}$. Cette démarche a été empruntée par beaucoup d'économistes et gestionnaires forestiers depuis plusieurs siècles et notamment les trois derniers. En dépit de son ancienneté, elle a suscité de nombreux débats discutant les bases sur lesquelles doit être assise cette optimisation, et plus concrètement sur la fonction objectif qu'il convient d'optimiser pour obtenir un tel résultat, ainsi que les contraintes qui viendront limiter le domaine des solutions réalisables. On appelle généralement « critère de gestion » toute expression traduisant la stratégie du propriétaire et étant appelée à figurer soit dans la fonction objectif, soit dans une contrainte.

Le problème soulevé dépasse en réalité celui de la seule détermination de l'âge d'exploitation et concerne plus largement les bases rationnelles sur lesquelles l'ensemble de la gestion des peuplements pourrait être conduite. Ainsi, non seulement l'âge d'exploitation doit-il être optimisé, mais également le calendrier, l'intensité, la nature des éclaircies ou encore la densité de plantation. De manière encore plus générale, les applications sont susceptibles de porter sur l'ensemble des investissements forestiers.

Ce type de question n'est pas à proprement parler spécifique au domaine forestier. Ce dernier possède cependant cer-

1. Peuplements dans lesquels tous les arbres ont le même âge ou à peu près le même âge.

2. Il est ici considéré que l'âge d'exploitabilité est l'âge optimal d'exploitation des peuplements taines particularités qui méritent d'être rappelées.

- La durée, tout d'abord, qui caractérise les investissements réalisés; elle rend parfois illusoire aux yeux des gestionnaires tout calcul économique précis à un horizon supérieur à leur propre espérance de vie ; elle induit cependant aussi des comportements particuliers comme celui d'une grande sensibilité à la régularité des interventions, à la nature soutenue des productions, au caractère durable de la gestion.

- La structure spécifique du flux de recettes d'un investissement ; contrairement au cas des investissements industriels pour lesquels la dépense initiale engendre immédiatement des recettes nettes modérées mais régulières dans le temps, les investissements forestiers produisent l'essentiel de leurs revenus tout à la fin de la révolution et à un niveau qui doit être très supérieur aux sommes investies.

- La difficulté, pour la majorité des sylvicultures pratiquées, de distinguer le capital productif du produit; elle n'est pas propre à la forêt (un cheptel d'animaux présente des caractéristiques semblables), mais n'existe pas dans le cas des investissements industriels typiques.

- L'incidence de multiples aléas qui affectent les arbres et les peuplements, et peuvent susciter des approches intégrant le risque et l'irréversibilité des choix.

- La multiplicité des produits forestiers, biens et services, marchands et non marchands, qui n'est pas le facteur le moins important; elle ne peut être prise en compte intégralement et justifie la multiplicité des critères utilisés qui seront donc présentés ici dans leur grande majorité et comparés les uns aux autres.

Les critères de gestion traditionnellement cités dans la littérature sont nombreux. Certains s'appuient par exemple sur de simples considérations physiques, rapportées à l'année ou non, d'autres sur 
les dépenses et revenus, d'autres encore sur la théorie des investissements. S'il existe des arguments forts plaidant pour certains de ces critères, aucun n'est véritablement incontestable car tous reposent sur des hypothèses strictes qui ne sont pas forcément respectées dans la réalité et qui seront plus adaptées à certaines situations qu'à d'autres. La considération de plusieurs d'entre ces critères reste donc utile pour le propriétaire ou le gestionnaire forestier qui pourra ainsi se déterminer par rapport à un ensemble de possibilités. La pratique montre d'ailleurs que les critères, lorsqu'ils sont explicites, diffèrent largement d'un propriétaire à l'autre.

Dans ce contexte, une discussion critique sur les mérites et les effets comparés de chacun des critères envisageables s'avère certainement utile et sans aucun doute susceptible d'éclairer les décideurs forestiers dans les différents choix qui s'offrent à eux. Si de nombreuses revues bibliographiques ont été produites sur le sujet, la plupart excellentes [17, 31, 34, $37,40-42,46]$, il s'est avéré possible encore de les compléter, de proposer une structuration des différents critères, de mettre en évidence les principales différences entre ces critères, et de préciser la terminologie en langue française.

Les deux premières sections se situeront dans un cadre théorique précis, qui sera caractérisé par les hypothèses suivantes.

- Le peuplement forestier considéré est équienne, et son âge possède ainsi une réelle signification ; cette hypothèse assure une bonne prise en compte du temps dont l'importance est considérable dans ce type de démarche ; une formulation continue de cet âge est utilisée, par souci de cohérence, en raison des dérivations à effectuer.

- Le raisonnement porte a priori sur le long terme, c'est-à-dire depuis la constitution initiale (naturelle ou artificielle) jusqu'à l'exploitation finale du peuplement.

- Les développements se réfèrent à un cas simplifié pour lequel les recettes et dépenses intermédiaires, annuelles ou occasionnelles, sont négligées ; les calculs mathématiques se trouvent simplifiés et certaines représentations graphiques sont rendues possibles.

- Les externalités (biens ou services non marchands) ne sont pas prises en compte, ni les productions connexes comme la chasse : la source essentielle de revenu est constituée par les ventes de bois.

- Le prix des bois est une fonction de l'âge, directe ou indirecte ; il dépend dans ce dernier cas d'une autre variable (grosseur moyenne à hauteur de poitrine, volume de l'arbre moyen,...) elle-même fonction monotone de l'âge.

- Le taux d'actualisation du décideur est unique et bien déterminé.

- L'avenir est certain et exempt de risques, à la fois quant à la croissance des peuplements et au contexte économique (prix des bois, coûts des travaux, taux d'intérêt).

Au cours des sections suivantes, la plupart de ces hypothèses seront levées une à une :

- en introduisant les dépenses et recettes intermédiaires de façon à se rapprocher des cas réels dans toute leur complexité ;

- en analysant le cas de forêts constituées de plusieurs peuplements équiennes, ou encore de peuplements inéquiennes, lorsqu'un objectif de recherche des rendements soutenus est poursuivi ;

- en appréciant comment les critères développés dans le long terme sont adaptables au cas des peuplements actuels qui sont dans une phase transitoire vers la récolte ou l'équilibre ;

- en développant le cas de marchés financiers imparfaits et en discutant la notion de taux d'actualisation. 


\section{APERÇU ANALYTIQUE DES PRINCIPAUX CRITERES DE GESTION}

La recherche d'une relative exhaustivité ne doit pas conduire à une trop grande complexité. C'est pourquoi les différents critères sont tout d'abord structurés en trois grands niveaux.

1. Les critères d'accumulation, qui considèrent que la forêt est un stock, que les terrains à boiser existent en quantités illimitées, et que la production est importante indépendamment du temps nécessaire à son obtention.

2. Les critères de productivité, qui prennent acte de la rareté des terrains forestiers, introduisent le facteur temps sans aller toutefois jusqu'à lui affecter une valeur, s'appuient sur les quantités produites, considèrent le cas échéant, sous une forme ou sous une autre, la valeur des produits, et éventuellement les dépenses consenties ; ils ont un caractère technicoéconomique.

3. Les critères de rentabilité, de nature économique et financière, qui donnent au temps une valeur représentée par un taux d'intérêt, de placement, d'actualisation ou de rentabilité interne, qui font référence aux investissements concurrents et donc au concept de coût d'opportunité de la solution retenue, qui tendent également à comparer le bénéfice retiré à terme d'un investissement à la dépense initialement consentie, ou encore les revenus engendrés par le peuplement à sa valeur sur pied.

Chaque critère est analysé à partir de son expression simplifiée, en âge continu. Il est alors possible de calculer la dérivée du critère simplifié par rapport à l'âge pour déterminer les conditions mathématiques du premier ordre correspondant à la maximisation du critère simplifié. Les conditions du deuxième ordre (négativité de la dérivée seconde qui assure que l'optimum est un maximum) ne sont pas explicitées pour ne pas alourdir la présentation.
Compte tenu du grand nombre de critères potentiels, chacun d'entre eux est désigné, non pas par un seul qualificatif, mais par un intitulé précis, auquel est associé un sigle. En effet, la terminologie qui a parfois été utilisée en France [31, 40, $42,46,47]$, sous des formes d'ailleurs variables selon les auteurs, qui distingue par exemple des exploitabilités culturale, physique, technique, absolue, relative, composée, économique, commerciale, financière, $s$ 'avère finalement peu précise, insuffisamment suggestive ${ }^{3}$, voire en contradiction avec la terminologie anglaise ${ }^{4}$.

Les notations utilisées sont ainsi les suivantes.

$a$ : âge du peuplement, variant de $o$ à $n$;

$D_{a}$ : dépenses annuelles par unité de surface à l'âge $a$;

$p_{a}$ : prix des bois relatif aux caractéristiques du peuplement à l'âge $a$, net des frais de marquage, mise en vente et contrôle de l'exploitation, par unité de volume ;

$V_{a}:$ volume exploité par unité de surface à l'âge $a$;

$R_{a}$ : revenus annuels encaissés par unité de surface à l'âge $a$;

$r$ : taux d'actualisation, lorsqu'il est choisi ;

$F$ : valeur, lorsqu'elle est fixée de façon exogène, du fonds forestier par unité de surface.

3. L'exploitabilité des économistes serait l'exploitabilité commerciale et non l'exploitabilité économique ! Le qualificatif « absolu» n'est pas très heureux car il renvoie à une attitude normative qu'il convient justement d'éviter.

4. Financial maturity est en anglais ce que les auteurs français ont appelé l'exploitabilité commerciale. 
Le revenu brut correspondant à un âge $a$ est en général présenté sous sa forme agrégée $R_{a}$, parfois décomposé en $p_{a} V_{a}$.

\subsection{Les critères d'accumulation}

\subsubsection{Considérations préliminaires}

On distingue généralement en économie la production totale physique de la production totale en valeur. La seconde se déduit de la première par l'adjonction d'un prix.

La production totale physique est communément considérée sous sa forme production totale en volume (VT), qui peut être définie comme le volume sur pied des arbres vivants du peuplement, augmenté du volume des éclaircies réalisées. On définirait de même une production totale en surface terrière, dont l'utilisation serait cependant moins fructueuse que la précédente dans la mesure où elle ne peut être directement valorisée. La production totale en masse n'a pas été utilisée à notre connaissance comme critère de gestion ; elle est cependant largement corrélée à la production en volume et pourrait correspondre à certains débouchés particuliers comme ceux de la trituration.

Dans le même esprit, la production totale en valeur se définit par la valeur mobilière du peuplement sur pied augmentée de la valeur des éclaircies et autres produits passés. Cette production totale est brute ou nette des coûts de plantation, de gestion, de garderie, d'imposition. Les recettes et dépenses sont évaluées sur la base des prix d'une année de référence, en général l'année des calculs. Elles ne sont pas exprimées ici en valeur actualisée. La production totale en valeur est dénommée revenu total $(R T)$ lorsqu'elle est prise sous forme brute, et bénéfice total (BT) lorsqu'elle est nette.

Comme on passe du volume au revenu par l'intermédiaire d'un prix, on pourrait de même multiplier la surface terrière par la « hauteur de forme » pour obtenir le volume, ou ce dernier par l'infradensité pour calculer la masse [33].

La présentation des critères d'accumulation s'appuie ici sur le cas du bénéfice total $B T$ dont on peut très facilement déduire le revenu total $R T$ en négligeant les coûts, puis le volume total $V T$ en raisonnant à prix constants (et égaux à une unité de prix).

\subsubsection{Bénéfice total (B'T)}

$$
B T=R_{n}-D_{\circ}
$$

Pour rendre maximal le bénéfice total, il suffit d'annuler la dérivée de son expression par rapport à l'âge $n$. On obtient alors :

$$
\frac{\mathrm{d} B T}{\mathrm{~d} n}=\frac{\mathrm{d} R_{n}}{\mathrm{~d} n}=0 \quad \text { avec } \quad R_{n}=p_{n} \cdot V_{n}
$$

soit encore :

$$
\frac{1}{p_{n}} \cdot \frac{\mathrm{d} p_{n}}{\mathrm{~d} n}=-\frac{1}{V_{n}} \cdot \frac{\mathrm{d} V_{n}}{\mathrm{~d} n}
$$

À l'optimum, les taux d'accroissement du prix et du volume sont donc opposés. C'est-à-dire que la diminution relative du prix est égale à l'augmentation relative du volume.

\subsubsection{Commentaires sur l'accumulation}

Ces critères d'accumulation conduisent à maximiser un capital plus qu'un produit. Ils auraient été utilisés jusqu'à une date relativement récente en Allemagne et dans d'autres pays (Thomson, 1942, cité par [34]). Ils sont proches, dans les faits, de stratégies de protection ou de récréation tendant à garder les peuplements sur pied le plus longtemps possible, en intervenant seulement en coupes sanitaires. Ils seraient ainsi voisins de l'idée d'exploitabilité dite physique, dans leur version en volume. $\mathrm{Si}$ 
les terrains forestiers existaient en quantité illimitée, ils pourraient traduire l'idée d'un stockage le plus long possible du carbone fixé par photosynthèse ; cependant, la plupart du temps, les terrains à reboiser ne sont pas indéfiniment disponibles.

\subsection{Les critères de productivité}

\subsubsection{Considérations préliminaires}

Il s'agit non plus de maximiser une production indépendamment du temps nécessaire à la réaliser, mais le flux annuel moyen correspondant, en particulier parce que la disponibilité de terrains pour la forêt est limitée. Comme précédemment, ce flux annuel peut être considéré pour plusieurs grandeurs.

La production moyenne annuelle en volume $V M$ est la mesure de productivité physique la plus communément utilisée. Elle se déduit directement de la production totale en volume $V T$.

En ce qui concerne la productivité en valeur, on associe le revenu moyen annuel $R M$ au revenu total $R T$ et le bénéfice moyen annuel $B M$ au bénéfice total $B T$. Comme précédemment, il est suffisant de se limiter à l'analyse plus précise d'un seul de ces critères, le bénéfice moyen annuel $B M$. De ce dernier, il est en effet aisé de déduire les autres comme cela a été indiqué pour les critères d'accumulation correspondants.

\subsubsection{Bénéfice moyen annuel (BM)}

$$
B M=\frac{B T}{n}=\frac{R_{n}-D_{o}}{n}=\frac{p_{n} \cdot V_{n}-D_{o}}{n}
$$

En dérivant ce bénéfice moyen par rapport à l'âge $n$, on obtient :

$$
\frac{\mathrm{d} B M}{\mathrm{~d} n}=\frac{1}{n} \cdot\left(\frac{\mathrm{d} B T}{\mathrm{~d} n}-\frac{B T}{n}\right)=\frac{1}{n} \cdot\left(\frac{\mathrm{d} R_{n}}{\mathrm{~d} n}-B M\right)
$$

L'optimum vérifie nécessairement les conditions équivalentes :

$$
\begin{gathered}
\frac{\mathrm{d} B T}{\mathrm{~d} n}=\frac{B T}{n}=B M \\
\text { et } \frac{\mathrm{d} R_{n}}{\mathrm{~d} n} \cdot \frac{1}{R_{n}-D_{n}}=\frac{1}{n}
\end{gathered}
$$

Lorsque le bénéfice moyen $B M$ est maximal, il est donc égal au bénéfice marginal $\frac{\mathrm{d} B T}{\mathrm{~d} n}$, résultat classique tant en foresterie qu'en économie. On peut aussi le formuler de la façon suivante : à l'optimum, le bénéfice total $B T$ s'accrô̂t à un rythme inversement proportionnel à l'âge du peuplement.

On note également que, si $B M^{*}$ désigne la valeur de $B M$ à l'optimum, alors la première forme des équations (6) entraîne que l'expression $B T-n . B M^{*}$ est négative sauf à l'optimum où elle culmine et s'annule.

\subsubsection{Commentaires sur la productivité}

Ces critères sont bien connus de tous les forestiers. Réaumur, vers 1721, Duhamel du Monceau, vers 1764, Buffon, vers 1774 , se sont intéressés à la plus grande productivité des bois, notamment de taillis. Dans son « Traité complet des bois et forêts », Duhamel du Monceau [11] préconise de couper les taillis lorsqu'ils fournissent le plus fort revenu moyen annuel (partie 1, livre 2, chapitre 1, article 2). Mais, selon Baudrillart [1], «l'auteur qui paraît avoir mis le plus d'exactitude dans ses recherches sur l'accroissement des bois [...] est Varenne de Fenille ». Ce dernier [47] fournit en particulier la méthode de détermination du moment où l'accroissement moyen en volume est maximal : il donne comme règle d'attendre l'égalité 
entre les deux accroissements courant et moyen ${ }^{5}$.

Assez facilement accessible, et très palpable, la productivité physique en volume $V M$ a souvent été préconisée. La loi forestière américaine de 1976 (National Forest Management $A c t$ ) l'impose pour les forêts nationales [34]. En France, l'Office national des forêts reconnaît sa valeur de référence $[37,38]$. Au niveau d'un pays dans lequel le stock de terrains disponibles pour la production forestière est limité, il correspond à une stratégie de maximisation de la quantité de bois produite, ou de carbone fixé (lorsque la masse anhydre est utilisée en lieu et place du volume).

Sa signification pour un propriétaire ou un gestionnaire forestier est cependant d'autant moins claire que la qualité des bois est hétérogène. L'affectation d'un poids plus grand aux bois de meilleure qualité, par exemple par l'intermédiaire d'un prix, apparaît alors nécessaire. On aborde ainsi les critères de productivité en valeur, qui ont d'ailleurs été conçus en tout premier lieu [11, 19]. En France, ces derniers semblent avoir implicitement la faveur de l'Office national des forêts [37]. Ils sont parfois appelés « rente forestière », ou « feuille ».

Le principal inconvénient des critères précédents est qu'ils ne prennent absolument pas en compte le coût de l'immobilisation des capitaux. Or, tout propriétaire peut toujours souhaiter financer un projet forestier plus productif plutôt qu'un autre (même l'État), et conduit en général aussi

5. Varenne de Fenille est considéré comme un inspirateur de Cotta [26] ; à la même époque, G.L. Hartig, qui a diffusé les techniques de coupe de régénération progressive et la conception actuelle de la sylviculture des futaies régulières, compare la productivité en volume de la futaie et du taillis sur une durée fixe de 120 ans correspondant à une révolution de futaie, et à 3 ou 4 révolutions de taillis [19]. d'autres projets non forestiers qui vont constituer autant d'options concurrentes. Ce qui amène à utiliser des critères de rentabilité.

\subsection{Les critères de rentabilité}

\subsubsection{Considérations préliminaires}

Contrairement aux deux groupes précédents, les critères de rentabilité constituent un ensemble beaucoup moins homogène au sein duquel plusieurs formulations principales différentes méritent d'être distinguées. Néanmoins, ils s'appuient tous sur un taux d'intérêt, qui pourra être un taux d'actualisation ou un taux interne de rentabilité, qui traduit la préférence pour le présent des agents, exprime les possibilités concurrentes de placement, fournit une mesure de la rentabilité. L'actualisation des bénéfices à un certain taux permet d'intégrer dans l'analyse le coût que représente le fait de renoncer à des placements ou prêts à ce taux. Elle prend donc en considération les charges financières occasionnées par la gestion forestière. Elle intègre ainsi l'ensemble des coûts du projet. C'est la raison pour laquelle elle traduit véritablement la méthode économique.

Les divers critères de rentabilité diffèrent entre eux sur les deux points suivants :

- la valeur du fonds est exogène (éventuellement négligée), ou endogène ;

- le taux d'actualisation est fixé ou révélé (et généralement assimilé, dans ce dernier cas, à un taux interne de rentabilité).

On utilise la seule formulation en temps continu en représentant le facteur d'actualisation sur $n$ années sous la forme exponentielle $\mathrm{e}^{-n r}$ plutôt que sous la puissance

$n^{\text {ième }}$ de $\frac{1}{1+r}$ soit $\frac{1}{(1+r)^{n}}$. 


\subsubsection{Bénéfice actualisé sur une seule révolution (BAS, BASF)}

Le bénéfice actualisé sur une seule révolution n'a d'autre signification que l'équivalent actuel du profit réalisé sur $n$ années. Il est noté $B A S$ lorsqu'il néglige la valeur du fonds, et $B A S F$ lorsque l'immobilisation du fonds est considérée comme un coût. C'est cette dernière formulation, plus générale, qui est développée ici :

$$
B A S F=-\left(F+D_{o}\right)+\left(F+R_{n}\right) \cdot \mathrm{e}^{-n r}
$$

La dérivée de ce bénéfice actualisé par rapport à l'âge d'exploitation $n$ s'écrit :

$$
\frac{\mathrm{d} B A S F}{\mathrm{~d} n}=\left(\frac{\mathrm{d} R_{n}}{\mathrm{~d} n}-r .\left(F+R_{n}\right)\right) \cdot \mathrm{e}^{-n r}
$$

La condition d'optimalité du premier ordre est alors :

$$
\begin{gathered}
\frac{\mathrm{d} R_{n}}{\mathrm{~d} n} \cdot \frac{1}{F+R_{n}}=r \\
\text { ou } \frac{\mathrm{d} R_{n}}{\mathrm{~d} n}=r \cdot\left(F+R_{n}\right)
\end{gathered}
$$

L'optimum est donc atteint lorsque la somme des valeurs du fonds et des bois qu'il porte s'accroît au taux $r$ ou encore lorsque le coût annuel d'immobilisation $r_{.}\left(F+R_{n}\right)$ équivaut au gain marginal $\frac{\mathrm{d} R_{n}}{\mathrm{~d} n}$.

\subsubsection{Bénéfice actualisé sur une infinité de révolutions (BASI)}

Le bénéfice actualisé sur une infinité de révolutions possède une signification beaucoup plus intéressante que le simple bénéfice actualisé sur une seule révolution. Il représente en effet la valeur du fonds logiquement calculée à partir du flux actualisé de recettes et de dépenses auquel ce dernier conduira $[12,13,14]$. Diverses formulations peuvent en être données :

$$
\begin{gathered}
B A S I=\frac{R_{n} \cdot \mathrm{e}^{-n r}-D_{o}}{1-\mathrm{e}^{-n r}} \\
=-D_{o}+\frac{R_{n}-D_{o}}{\mathrm{e}^{n r}-1}=-R_{n}+\frac{R_{n} \cdot-D_{o}}{1-\mathrm{e}^{-n r}}
\end{gathered}
$$

ou encore :

$$
\left(B A S I+D_{0}\right) \cdot \mathrm{e}^{m}=B A S I+R_{n}
$$

Dérivons ce bénéfice actualisé par rapport à l'âge $n$; on obtient :

$$
\frac{\mathrm{d} B A S I}{\mathrm{~d} n}=\frac{1}{\mathrm{e}^{m r}-1} \cdot\left(\frac{\mathrm{d} R_{n}}{\mathrm{~d} n}-r \cdot \mathrm{e}^{n r} \cdot\left(B A S I+D_{0}\right)\right)
$$

La condition d'optimalité du premier ordre s'écrit alors sous l'une quelconque des formes suivantes :

$$
\left\{\begin{array}{l}
\frac{\mathrm{d} R_{n}}{\mathrm{~d} n}=r \cdot\left(B A S I+R_{n}\right)=r \cdot \frac{R_{n}-D_{t}}{1-\mathrm{e}^{-n r}} \\
\text { ou } \frac{\mathrm{d} R_{n}}{\mathrm{~d} n} \cdot \frac{1}{B A S I+R_{n}}=r \\
\text { ou bien encore } \\
\frac{\mathrm{d} R_{n}}{\mathrm{~d} n} \cdot \frac{1}{R_{n}-D_{n}}=\frac{r}{1-\mathrm{e}^{-n r}}
\end{array}\right.
$$

On retrouve le résultat précédent, à savoir que l'optimum est atteint lorsque la somme des valeurs du fonds et des bois qu'il porte s'accrô̂t au taux $r$ et que le coût d'immobilisation $\frac{\mathrm{d} R_{n}}{\mathrm{~d} n}$ est égal au gain marginal $r .\left(B A S I+R_{n}\right)$. La seule différence est ici que la valeur du fonds est optimisée.

\subsubsection{Taux interne de rentabilité (TIR, TIRF)}

Un taux interne de rentabilité annule un bénéfice actualisé. Il apparaît ainsi clai- 
rement comme le taux auquel l'argent investi a fonctionné puisque alors les revenus actualisés à ce taux sont égaux aux dépenses initiales ou, ce qui revient au même, les dépenses capitalisées à ce taux sont égales aux revenus finaux. En correspondance avec les deux bénéfices actualisés distingués, $B A S$ et $B A S F$, deux taux internes de rentabilité peuvent être définis, l'un négligeant la valeur du fonds $(T I R)$, l'autre l'incluant (TIRF). C'est encore une fois la formulation la plus générale (TIRF) qui est présentée ici.

Le cas simplifié correspond à l'une des rares occasions dans lesquelles le taux interne de rentabilité peut être explicité :

$$
\left\{\begin{array}{l}
\text { TIRF }=\frac{1}{n} \cdot \log \left(\frac{F+R_{n}}{F+D_{o}}\right) \\
R_{n}=\left(F+D_{o}\right) \cdot \mathrm{e}^{n \cdot T I R F}-F
\end{array}\right.
$$

En dérivant ce taux par rapport à l'âge $n$, on obtient :

$$
\frac{\mathrm{d} T I R F}{\mathrm{~d} n}=\frac{1}{n \cdot\left(F+R_{n}\right)} \cdot\left(\frac{\mathrm{d} R_{n}}{\mathrm{~d} n}-\left(F+R_{n}\right) \cdot T I R F\right)
$$

La condition d'optimalité du premier ordre s'écrit alors :

$$
\frac{\mathrm{d} R_{n}}{\mathrm{~d} n} \cdot \frac{1}{F+R_{n}}=T I R F
$$

On trouve une nouvelle fois les résultats précédents, à savoir que l'optimum est atteint lorsque la somme des valeurs du fonds et des bois qu'il porte s'accroît au taux qui est ici le maximum du taux interne de rentabilité. En revanche, si $\frac{\mathrm{d} R_{n}}{\mathrm{~d} n}$ représente toujours le revenu marginal TIRF $\cdot\left(F+R_{n}\right)$, n'est plus le coût marginal de l'immobilisation, la valeur de cette dernière devant être comptée au taux d'actualisation, et non au taux interne de rentabilité.

\subsection{Commentaires sur la rentabilité}

Les critères de rentabilité définis ici sont donc au nombre de cinq : $B A S, B A S F$, $B A S I, T I R, T I R F$. Ils prennent tous en compte le coût financier associé à la durée des projets forestiers. Ils ont par ailleurs de nombreuses relations entre eux. On peut remarquer en effet que le $T I R$ annule le $B A S$ et donc aussi nécessairement le $B A S I$. De même, annulant le $B A S F$, le TIRF rend le BASI égal à $F$.

Les critères excluant la valeur du fonds (BAS,TIR) supposent le fonds de valeur nulle, c'est-à-dire inaliénable ou présent en quantité illimitée. L'inaliénabilité de terrains forestiers ne peut être garantie : une forêt soumise au régime forestier peut en être distraite et les boisements qui se formeraient sur le domaine public, c'est-àdire en pratique sur des relais de mer abandonnés par les eaux, sont susceptibles de faire l'objet d'un reclassement au profit du domaine privé de l'État [2]. Quant au caractère illimité des terrains à boiser, il est rare qu'il puisse être pris pour hypothèse.

Les critères assis sur une seule révolution ont été proposés à l'origine par des économistes reconnus comme Wicksell et Von Thünen [23]. Mais ils ont été dénigrés par Samuelson [41] ; ils ne prennent en considération la répercussion de la durée de production sur les revenus ultérieurs de la forêt qu'à la condition d'introduire la valeur du fonds de façon exogène $(B A S F)$. Investie l'année 0 , cette valeur est rendue à nouveau disponible à la fin de la révolution. Son équivalent actuel est d'autant plus petit que la révolution s'allonge, et le coût sur les révolutions ultérieures d'un tel allongement se trouve ainsi comptabilisé [41].

La notion de taux interne de rentabilité a été introduite en économie par Fisher en 1907, et préconisée en foresterie par Chapman en $1915[6,16]$, et plus récemment par Hiley en 1930 [20] et Boulding en 1955 [34]. De nombreuses objections ont 
cependant été formulées à son encontre, notamment en ce qui concerne la maximisation de la formulation qui néglige la valeur du fonds. En effet, lorsque la quantité de terrains à boiser est limitée et que le propriétaire possède des disponibilités financières ou peut les emprunter, maximiser le taux $T I R$ ne revient pas à maximiser un profit. Ce qui a conduit de nombreux auteurs à prendre leurs distances avec la maximisation du taux interne de rentabilité $[15,34,41]$. En revanche, le taux TIRF peut être considéré comme un révélateur du taux d'actualisation utilisé par les agents, à la condition toutefois que la valeur exogène du fonds soit adéquate. Alors, maximiser le $B A S I$ pour un taux égal au $T I R F$ revient à maximiser le $T I R F$ pour une valeur du fonds égale au $B A S I$ [41].

Assis sur une infinité de révolutions, le critère du $B A S I$, est le plus conforme à la théorie micro-économique $[10,12-15$,
$17,23,25,30,41,43-45]$. La formule (10), exprimée sous trois formes différentes, équivalente à la formule (11) et dite formule de Faustmann, a été établie en réalité par König, en 1813, [25, 43] ; c'est cependant à Faustmann que revient le mérite d'avoir clairement montré son intérêt pour l'optimisation de la gestion forestière ${ }^{6}$.

\section{ANALYSE COMPARATIVE DES DIFFÉRENTS CRITÈRES SIMPLIFIÉS}

Les hypothèses générales restent celles de la section précédente pour récapituler les différents critères qui viennent d'être présentés, ainsi que ceux qui s'en déduisent aisément. Ils sont au nombre de 11 . C'est dire combien il apparaît nécessaire de les structurer et de leur donner une dénomination qui puisse servir de réfé-

\begin{abstract}
6. Après König, Cotta [7,8] produit les tables permettant de mettre aisément en œuvre la formule du fonds. Mais ce dernier a du mal à admettre qu'il convient dans tous les cas d'utiliser des intérêts composés qui nécessitent la simultanéité entre une recette et son réinvestissement, ce qui lui apparaît irréaliste ; de la même façon, les intérêts simples signifieraient qu'aucune recette n'est réinvestie, ce qui ne lui semble pas meilleur ; il préconise donc d'utiliser un compromis entre intérêts simples et composés, puisque la réalité se situe entre ces deux extrêmes. Une telle ambiguïté sera abandonnée par la suite au profit des seuls intérêts composés. Plus tard, Faustmann [12] publie dans Allgemeine Forst- und Jagdzeitung une application de cette théorie. Celle-ci semble incorrecte à Von Gehren qui réplique la même année dans la même revue en arguant en particulier du fait que la valeur du fonds (le $B A S I$ ) s'avère négative dans un exemple qu'il a choisi [ 48 , 49]. Alors Faustmann $[13,14]$ est amené, en réponse, à développer à nouveau l'ensemble de la théorie qui le rendra finalement célèbre. Il montre ainsi que cette théorie permet tout à la fois de calculer la valeur du fonds (le $B A S I$ ), la valeur en bloc de la forêt, et d'optimiser la gestion forestière. Ce faisant, il corrige deux erreurs de Von Gehren : celle d'avoir pris l'exemple d'une sylviculture très conservatrice et donc non rentable (d'où la valeur négative du $B A S I$ ), l'autre d'avoir valorisé le peuplement à sa valeur de consommation et non à sa valeur d'avenir. Cette anecdote a été relatée par Gane [16], mais celui-ci n'a nulle part mentionné le premier article de Faustmann; il est vrai que ce dernier est signé F. et non «Faustmann » ; lorsqu'on sait cependant que Faustmann collaborait à la revue dont le rédacteur en Chef était l'Oberforstrat G.W. von Wedekind [29], il semble évident que cette signature ne peut être que la sienne. Le tort de Faustmann est peut-être de n'avoir pas perçu la portée de sa propre théorie au-delà du monde forestier et de ne pas l'avoir divulguée auprès des économistes de son époque. C'est pourquoi on considère souvent aujourd'hui que la théorie du calcul de la valeur d'un capital n'a été établie que vers 1900, grâce aux travaux de Boehm-Bawerk et de Fisher [16] et après le caractère précurseur de ceux, datés de 1834, de Mountiford Longfield et John Rae [9]. Ainsi, alors que Faustmann n'est pas cité dans les écrits économiques traitant de ce domaine, il est sans doute celui qui en a le plus tôt élaboré la théorie la plus complète, en 1849, après les premières approches de König en 1813.
\end{abstract}


rence. Ces deux aspects font l'objet du tableau I. Les trois grandes catégories d'accumulation, de productivité et de rentabilité y sont distinguées, ainsi que la nature de la variable optimisée : surface terrière, volume, revenu ou bénéfice. Enfin, les critères de rentabilité fondés sur les bénéfices font l'objet d'une double distinction suivant qu'ils fonctionnent sur une révolution, sur une infinité de révolutions, ou sur un nombre quelconque de révolutions, d'une part, et selon qu'ils négligent ou non le fonds, d'autre part.

Le choix des sigles a été fait de la façon suivante :

- la première lettre rend compte de la variable de base : volume (V), revenu $(R)$, taux d'intérêt (T) et bénéfice (B) qui a été préféré à revenu net; on s'appuierait de même sur la masse $(\mathrm{M})$, la surface terrière $(\mathrm{G})$ ou d'autres variables ;

- la seconde lettre indique le caractère total (T) ou moyen annuel (M) de la variable des critères d'accumulation et de productivité, le caractère actualisé (A) ou interne (I) des critères de rentabilité ;

- les sigles des critères d'accumulation et de productivité ont deux lettres ; ceux des critères de rentabilité en ont 3 ou 4 suivant que le fonds est exclu ou inclus; lorsque le même critère existe sans ou avec considération du fonds, un F marque ce dernier cas.

Le tableau I récapitule par ailleurs les principales caractéristiques des différents critères et synthétise ainsi les commentaires précédents.

Tableau I. Présentation structurée des différents critères de gestion applicables à un peuplement équienne considéré à long terme dans le cas simplifié où les dépenses et recettes intermédiaires sont négligées.

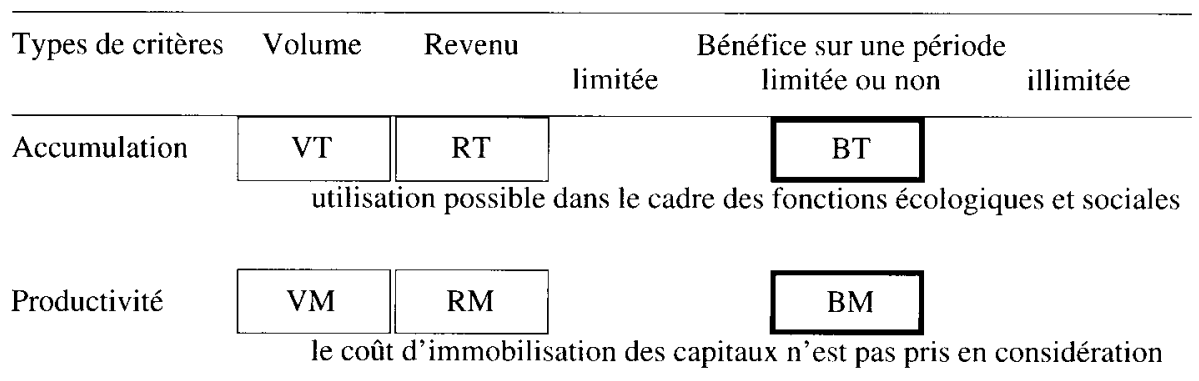

Rentabilité partielle

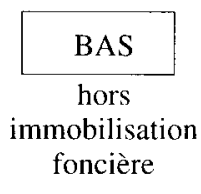

Rentabilité globale

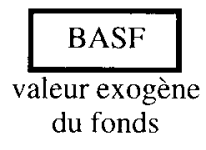

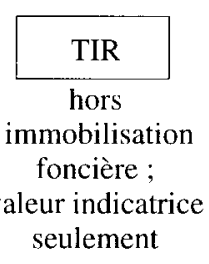

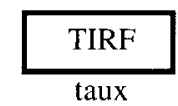

d'actualisation

révélé ou valeur indicatrice
BASI

valeur endogène

du fonds, critère de Faustmann 
La présentation qui vient d'être faite au travers de cinq formulations principales (encadrées en traits épais dans le tableau I) permet maintenant de tenter d'établir quelques règles prévalant lors de la comparaison des résultats obtenus selon l'un ou l'autre des critères. Dans le cas simplifié développé jusqu'à maintenant, ces résultats portent sur le problème classique de la révolution optimale. Les règles en question ne sont absolues que dans le cas simplifié, mais gardent une forte probabilité de rester approximativement valables dans les cas plus compliqués, comme cela pourra être vérifié par la suite. La comparaison entre les résultats des différents critères s'appuie ici sur trois techniques successives :

1. l'analyse des liaisons formelles entre certains critères ;

2. des comparaisons dans le plan (âge, revenu);

3. des comparaisons dans le plan (âge, taux d'accroissement).

\subsection{Comparaison entre critères liés par une fonction multiplicative}

La multiplication du volume récolté par un prix fournit un revenu. $V T$ et $R T$ d'une part, $V M$ et $R M$ d'autre part, sont ainsi liés par une fonction multiplicative, le prix. De façon analogue, les critères moyens $V M, R M$, et $B M$ sont liés aux critères totaux correspondants $V T, R T$ et $B T$ par l'intermédiaire de la fonction inverse de l'âge.

Le maximum d'un critère est noté $X^{*}$, et l'âge correspondant $n\left(X^{*}\right)$.

Compte tenu des règles de dérivation d'un produit, du caractère généralement encore croissant du prix lorsque culmine la production moyenne annuelle en volume, et décroissant lorsque le peuplement va dépérir, compte tenu enfin de la décrois- sance de la fonction inverse de l'âge, on peut affirmer que :

$$
\left\{\begin{array}{l}
n\left(V M^{*}\right) \leq n\left(R M^{*}\right) \leq n\left(R T^{*}\right)=n\left(B T^{*}\right) \leq n\left(V T^{*}\right) \\
n\left(B M^{*}\right) \leq n\left(B T^{*}\right)
\end{array}\right.
$$

Ainsi, les critères d'accumulation ( $V T$, $B T, R T$ ) tendent naturellement à retarder, en général considérablement, l'âge optimal d'exploitation. Par ailleurs, l'optimisation de la production moyenne en volume $(V M)$ conduit à une exploitation plus précoce que celle du revenu moyen annuel $(R M)$.

\subsection{Comparaison entre critères sur la base du revenu total $R T=R_{n}$}

Lorsqu' on se restreint aux critères reposant sur la notion de revenu ou de bénéfice (quatre dernières colonnes du tableau $I$ ), l'optimum peut être représenté dans le plan (âge, revenu) par rapport à la courbe d'évolution de $R_{n}$ selon l'âge. Les valeurs optimales de $R T$ et $B T$ sont par exemple naturellement atteintes au sommet de la courbe $R^{n}$, lorsque la tangente à la courbe est horizontale (figure l).

Le bénéfice moyen annuel est

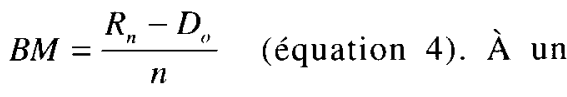
moment quelconque représenté sur la figure 1 par le point $M$, il correspond à la pente de la droite $D_{o} M$ puisque les coordonnées de $M$ sont respectivement l'âge $n$ et le bénéfice total. En déplaçant $M$ sur la courbe $R_{n}$, on voit que cette pente devient maximale lorsque la droite $D_{o} M$ est tangente à la courbe $R_{n}$. De la même façon, le revenu moyen annuel $R M$ culmine lorsque la droite $O M$ est tangente à la courbe $R_{n}$. Il est clair, graphiquement, que cet optimum ne peut qu'être plus précoce que celui du bénéfice moyen annuel dès 


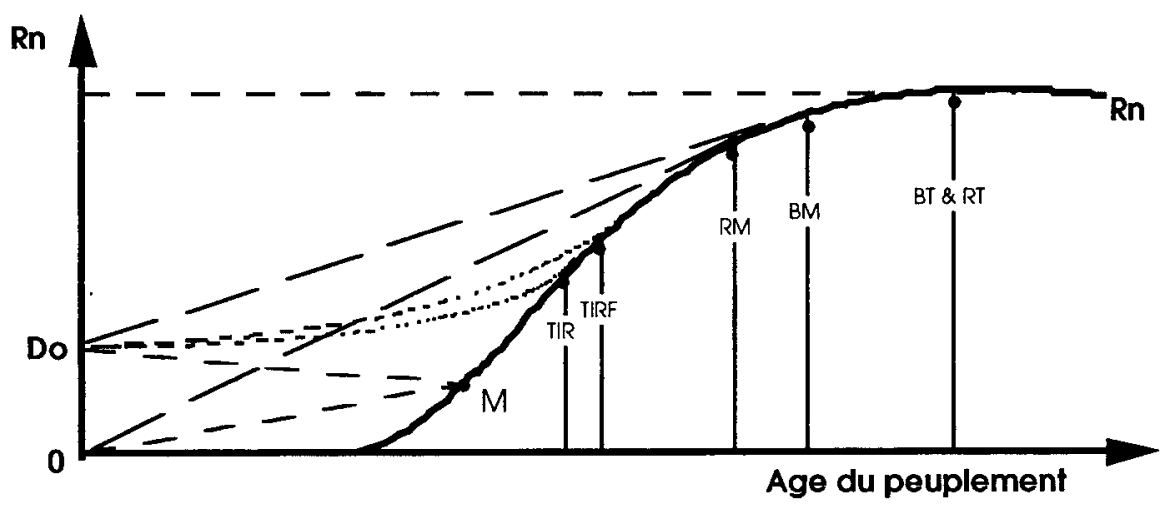

Figure 1. Comparaison dans le plan (âge, revenu) entre les âges optimaux d'exploitation découlant des critères $R T, B T, R M, B M, T I R$ ET TIRF.

lors que $D_{o}$ constitue une dépense effective et s'avère donc réellement positive. Si elle reste en outre modérée et surtout inférieure au maximum de $R_{n}$, ce qu'il faut espérer, alors :

$n\left(R M^{*}\right) \leq n\left(B M^{*}\right) \leq n\left(B T^{*}\right) \leq n\left(R T^{*}\right)$

Le taux interne de rentabilité TIRF est caractérisé par $R_{n}=\left(F+D_{o}\right) \cdot \mathrm{e}^{n \cdot T I R F}-F$ (équation 14). Il correspond donc au taux de croissance d'une exponentielle partant de $D_{o}$ et joignant la courbe $R_{n}$. Il est ainsi maximal lorsque l'exponentielle est tangente à la courbe $R^{n}$ comme le montre la figure 1 .

Le taux TIR constitue un cas particulier du taux TIRF lorsque $F$ est nul. on démontre aisément que la positivité de $F$ rend nécessairement $T I R F$ plus petit que $T I R$, et que la pente à l'origine de l'exponentielle de taux TIRF est plus grande que celle de l'exponentielle de taux TIR. L'exponentielle qui détermine $T I R F^{*}$ est donc plus inclinée au départ et plus tendue que celle qui détermine $T I R^{*}$ (figure 1 ). On en déduit facilement que :

$$
n\left(T I R^{*}\right) \leq n\left(T I R F^{*}\right)
$$

Il apparaît en outre que :

$$
n\left(T I R F^{*}\right) \leq n\left(B M^{*}\right)
$$

En revanche, la comparaison entre $T I R$ et $R M$ ne permet pas de conclure définitivement sur l'ordre dans lequel se présenteront les optimums correspondants. Si $D_{o}$ est particulièrement grande, l'âge d'obtention de $T I R^{*}$ pourrait être supérieur à celui de $R M^{*}$. En général cependant, c'est le cas de la figure 1 qui est réalisé.

Grâce à l'équation (7), le bénéfice actualisé sur une révolution est caractérisé par :

$$
R_{n}=\left(B A S F+F+D_{o}\right) \cdot \mathrm{e}^{n r}-F
$$

Son maximum se déduit donc aussi de la tangence à la courbe $R_{n}$ d'une exponentielle.

La comparaison entre $B A S F$ et $B A S$ montre immédiatement que :

$$
B A S-B A S F=F .\left(1-\mathrm{e}^{-n r}\right) \geq 0
$$

c'est-à-dire que : $B A S^{*} \geq B A S F^{*}$ 
De plus, la courbe exponentielle relative à $B A S$ est caractérisée par une ordonnée à l'origine supérieure et une pente initiale inférieure à celle de BASF (figure 2). On en tire donc:

$$
n\left(B A S F^{*}\right) \leq n\left(B A S^{*}\right)
$$

On voit également sur la figure 2 que la comparaison entre $B A S$ ou $B A S F$ et $B M$ ne permet pas toujours de conclure quant à l'ordre dans lequel interviendront les optimums correspondants. Si BASF ${ }^{*}$ et $B A S^{*}$ sont particulièrement grands ou, de manière équivalente, si $r$ est petit, alors l'optimum de $B M$ sera plus précoce que celui des deux autres critères. En effet, ces derniers tendent vers $B T$ quand le taux d'actualisation $r$ tend à s'annuler. Dans ce cas, l'actualisation n'induit donc pas obligatoirement une récolte plus précoce que la maximisation du bénéfice moyen annuel. En revanche, si $B A S F^{*}$ et $B A S^{*}$ sont petits, voire négatifs, la situation sera inversée.

Il reste maintenant à comparer ces bénéfices actualisées aux taux internes de rentabilité et à intégrer le $B A S I$ dans cette démarche. Entre ces cinq critères, les seuls résultats intangibles sont ceux des équations $(20)$ et $(21)$. Il est néanmoins possible, en distinguant les principaux cas de figure, de faire apparaître quelques résultats complémentaires.

L'équation (11) peut être réécrite sous la forme

$$
R_{n}=\left(B A S I+D_{o}\right) \cdot\left(1-\mathrm{e}^{-n r}\right)+D_{o}
$$

qui montre que le $B A S I$ peut aussi être illustré par la tangence entre la courbe $R_{n}$ et une courbe fondée sur une exponentielle d'ordonnée à l'origine $D_{o}$. Dans le cas particulier où $B A S F^{*}=0$, on a $T I R F^{*}=r$ et $B A S I^{*}=F$. En utilisant les équations (9), (13) et (16), on démontre immédiatement que ces optimums interviennent simultanément. De même, dans le cas particulier où $B A S^{*}=0$, on a $T I R^{*}=r$, $B A S I^{*}=0$ et les optimums correspondants sont simultanés. On débouche ainsi sur deux grands cas extrêmes dont les frontières viennent d'être délimitées :

- le premier est le cas rentable selon tous les critères, où $B A S F^{*}>0$, TIRF $^{*}>r, B A S I^{*}>F$, et donc aussi $B A S^{*}>0, T I R^{*}>r$; ce cas est celui de la figure 3 ;

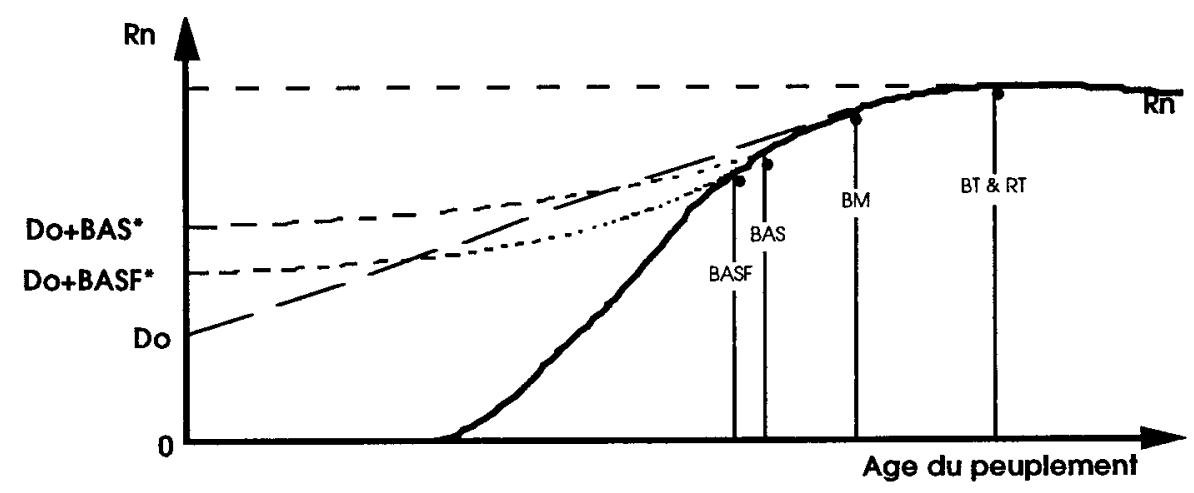

Figure 2. Comparaison dans le plan (âge, revenu) entre les âges optimaux d'exploitation découlant des critères $B M, B A S$ ET $B A S F$. 
- le second est le cas non rentable selon tous les critères,

$$
\text { où } B A S^{*}<0, T I R^{*}<r, B A S I^{*}<0,
$$

et donc aussi $B A S F^{*}<0, T I R F^{*}<r$; ce cas est celui de la figure 4 .

Entre ces deux extrêmes, la situation est plus complexe et offre toutes les transitions possibles.

\subsection{Comparaison entre critères sur la base des taux d'accroissement du revenu}

La représentation précédente dans le plan (âge, revenu) est relativement simple, mais les optimums y apparaissent sous la forme d'une tangence entre courbes, qui devient rapidement peu discernable dès que le nombre d'optimums dépasse quatre ou cinq. En raisonnant sur les taux

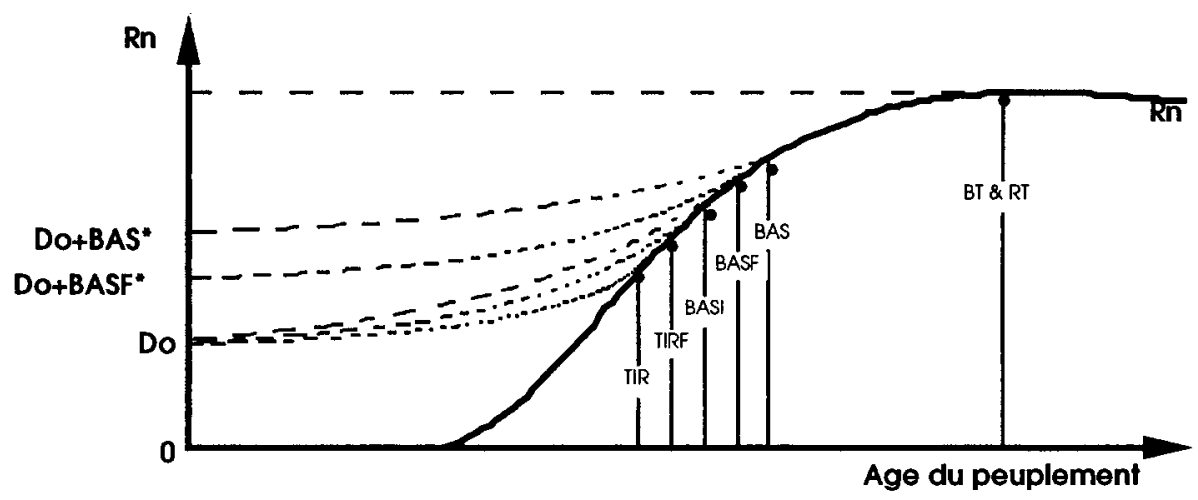

Figure 3. Comparaison dans le plan (âge, revenu) entre les âges optimaux d'exploitation découlant des critères $B A S, B A S F, B A S I, T I R$ ET TIRF (cas rentable selon tous les critères).

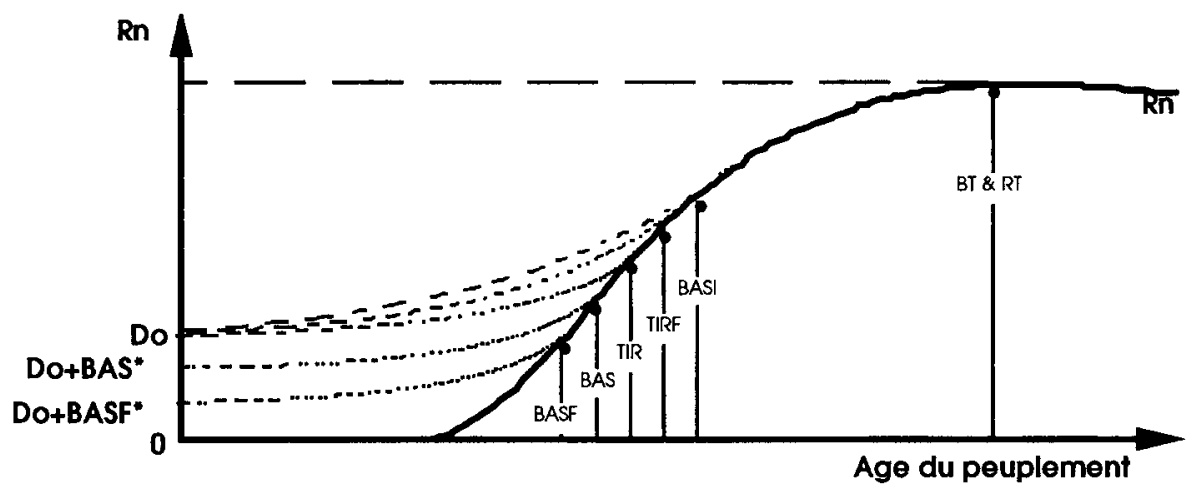

Figure 4. Comparaison dans le plan (âge, revenu) entre les âges optimaux d'exploitation découlant des critères $B A S, B A S F, B A S I, T I R$ ET TIRF (cas non rentable selon tous les critères). 
d'accroissement, les optimums se trouvent à l'intersection de courbes et la représentation devient moins confuse. La figure 5 permet ainsi de caractériser simultanément les neuf critères $R T, B T, R M$, $B M, B A S, B A S F, T I R, T I R F$ et BASI à partir des équations (3), (6), (9), (13) et (16). Elle complète la figure proposée par Newman [34].

Par exemple, en vertu de l'équation 13, le $B A S I$ est maximal pour $\frac{\mathrm{d} R_{n}}{\mathrm{~d} n} \cdot \frac{1}{R_{n}-D_{o}}=\frac{r}{1-\mathrm{e}^{-n r}}$. Les courbes de $\frac{\mathrm{d} R_{n}}{\mathrm{~d} n} \cdot \frac{1}{R_{n}-D_{o}}$ et $\frac{r}{1-\mathrm{e}^{-n r}}$ en fonction de $n$ apparaissent sur la figure 5 et leur intersection caractérise le $B A S I^{*}$. Il apparaît en particulier clairement sur la figure 5 que, lorsqu'on fait varier à la hausse le taux $r$ d'actualisation, seules les courbes $r$ et $\frac{r}{1-\mathrm{e}^{-n r}}$ se déplacent. Cette dernière part de la courbe $\frac{1}{n}$ pour $r$ tendant vers 0 , et s'élève tout en restant constamment décroissante, si bien que l'âge d'exploitabilité relatif au BASI est d'abord supérieur puis inférieur à celui du $T I R F$. De même pour le BASI et le TIR. On voit aussi une nette différence de nature entre le $B A S I$ d'une part, les $B A S$ et $B A S F$ d'autre part. Lorsque le taux d'actualisation tend à s'annuler, l'optimum correspondant au premier critère tend vers celui de $B M$, tandis que l'optimum de chacun des deux autres tend vers celui de $B T$ et $R T$.

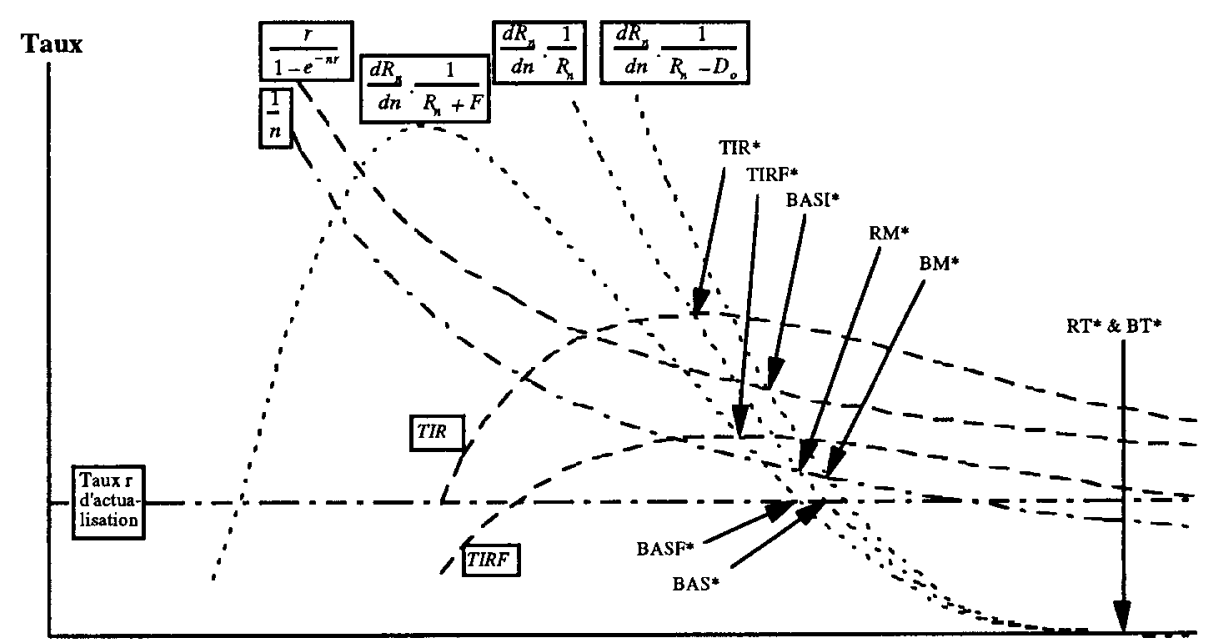

Age du peuplement

Figure 5. Comparaison dans le plan (âge, taux d'accroissement) entre les âges optimaux d'exploitation découlant des critères $R T, B T, R M, B M, B A S, B A S F, B A S I, T I R$ ET TIRF (ici dans le cas rentable pour tous les critères). 


\section{PRISE EN COMPTE DES DÉPENSES ET RECETTES INTERMÉDIAIRES}

Lorsque des dépenses et recettes intermédiaires sont prises en compte, les critères qui ont été présentés jusque là sous leur forme simplifiée changent peu. Dans la mesure où ces dépenses et recettes présentent souvent un caractère occasionnel, la formulation en temps continu qui a été utilisée dans le cas simplifié pour faciliter la dérivation ne convient plus. Elle est remplacée ci-après par une formulation en temps discret s'étendant sur $n$ années comprises entre les âges o (constitution du peuplement) et $n$ (exploitation) ${ }^{7}$.

\section{Critères d'accumulation}

$$
B T=\sum_{a=\rho}^{a=n}\left(R_{a}-D_{a}\right)
$$

\section{Critères de productivité}

$$
B M=\sum_{a=n}^{a=n} \frac{R_{a}-D_{a}}{n}
$$

\section{Critères de rentabilité}

$$
B A S F=\left(\sum_{a=n}^{a=n} \frac{R_{a}-D_{a}}{(1+r)^{a}}\right)-F \cdot \frac{(1+r)^{n}-1}{(1+r)^{n}}
$$

$T I R F$ en tant que racine de l'équation :

$$
F=\sum_{a=o}^{a=n} \frac{\left(R_{a}-D_{a}\right) \cdot(1+T I R F)^{n-a}}{(1+T I R F)^{n}-1}
$$

7. Bien que les sommes comprennent $(n+1)$ termes, elles portent sur $n$ années.

$$
B A S I=\sum_{a=o}^{a=n} \frac{\left(R_{a}-D_{a}\right) \cdot(1+r)^{n-a}}{(1+r)^{n}-1}
$$

Sous cette forme plus complète, la sylviculture à long terme ne se résume plus à la constitution initiale et à la récolte finale du peuplement. Elle comprend également des éclaircies dont on peut chercher à optimiser le calendrier, l'intensité, ou la nature. La résolution de ce type de problème est plus complexe et la meilleure méthode d'analyse est finalement celle qui repose sur des exemples qui permettent de vérifier dans quelle mesure subsistent les principes généraux établis dans le cas simplifié. Deux exemples sont ici succinctement étudiés, l'un relatif au pin maritime dans les landes de Gascogne, l'autre à l'épicéa commun dans le nord-est de la France. Les hypothèses relatives à chacun de ces exemples sont résumées dans le tableau II.

Les hypothèses du tableau II ont été intégrées dans le logiciel Sylopt élaboré par Guo [18] à partir de la méthode d'optimisation appelée «programmation dynamique ». L'avènement des optimums des critères d'accumulation sort en général du domaine de validité des modèles de croissance $^{8}$. Ainsi seuls ont été optimisés ici des critères de productivité et de rentabilité parmi lesquels ont été sélectionnés : $V M, B M$ et $B A S I$. Les résultats font l'objet des tableaux $I I I$ et $I V$ ainsi que des figures 6 et 7 . Ils sont évidemment représentatifs des hypothèses qui les sous-tendent et ne doivent pas être généralisés ; ils illustrent ce que peut être un résultat d'optimisation de l'ensemble du processus

8. Les critères d'accumulation sont optimaux au voisinage de ce que les forestiers appellent l'exploitabilité physique, c'est-à-dire la limite du dépérissement. Hormis dans les forêts non gérées, il est extrêmement rare que cette limite soit atteinte, si bien que les placettes servant à établir les modèles de croissance ont un âge qui est bien en deçà de cette limite. 
Tableau II. Principales hypothèses des deux exemples traités.

\begin{tabular}{|c|c|c|}
\hline Base des hypothèses & $\begin{array}{c}\text { Pin maritime dans les landes } \\
\text { de Gascogne }\end{array}$ & $\begin{array}{l}\text { Épicéa commun dans } \\
\text { le nord-est de la France }\end{array}$ \\
\hline Modèle de croissance & Lemoine [27] & Houllier [21] \\
\hline Hauteur dominante & $26,5 \mathrm{~m}$ à 40 ans & $25 \mathrm{~m}$ à 40 ans \\
\hline Taux d'actualisation (si préfixé) & $\begin{array}{l}29,3 \mathrm{~m} \text { a } 50 \text { ans } \\
3 \%\end{array}$ & $\begin{array}{l}30 \mathrm{~m} \text { a } 50 \text { ans } \\
3 \%\end{array}$ \\
\hline Valeur du fonds (si exogène) & $7500 \mathrm{~F} / \mathrm{ha}$ & $7500 \mathrm{~F} / \mathrm{ha}$ \\
\hline Coût de constitution & $10000 \mathrm{~F} / \mathrm{ha}$ & $\begin{array}{l}14144 \mathrm{~F} / \mathrm{ha} \\
13800 \mathrm{~F} \text { actualisés/ha }\end{array}$ \\
\hline Age initial & 11 ans & 20 ans \\
\hline Densité initiale & 1275 tiges $/ \mathrm{ha}$ & 1800 tiges/ha \\
\hline Surface terrière initiale & $16,7 \mathrm{~m}^{2} / \mathrm{ha}$ & $24 \mathrm{~m}^{2} / \mathrm{ha}$ \\
\hline Impôts fonciers & $0 \mathrm{~F} / \mathrm{an}$ & $\begin{array}{l}50 \mathrm{~F} / \mathrm{an} \\
\text { Exonération trentenaire }\end{array}$ \\
\hline Frais de gestion & $250 \mathrm{~F} / \mathrm{ha} / \mathrm{an}$ & $40 \mathrm{~F} / \mathrm{ha} / \mathrm{an}$ \\
\hline $\begin{array}{l}\text { Coût de martelage, mise } \\
\text { en vente et contrôle }\end{array}$ & $\begin{array}{l}350 \text { F/éclaircie } \\
450 \text { F/coupe rase }\end{array}$ & $\begin{array}{l}\text { éclaircie : } 22,0 \mathrm{~F} / \mathrm{m}^{3} \\
\text { coupe rase }: 24.2 \mathrm{~F} / \mathrm{m}^{3}\end{array}$ \\
\hline $\begin{array}{l}\text { Prix des bois en fonction du } \\
\text { volume v de l'arbre moyen }\left(\mathrm{F} / \mathrm{m}^{3}\right)\end{array}$ & $\begin{array}{l}\text { éclaircies : } 148 \cdot v^{0,451} \\
\text { coupe rase }: 184 . v^{0,633}\end{array}$ & $321 \cdot\left(1-\mathrm{e}^{-1,17 \cdot(v-0,1)}\right)$ \\
\hline $\begin{array}{l}\text { Contraintes sylvicoles imposées } \\
\text { aux facteurs ci-contre }\end{array}$ & $\begin{array}{l}\text { - intensité maximale des } \\
\text { éclaircies : } 40 \% \\
\text { - rotation minimale : } 3 \text { ans } \\
\text { - prélèvement minimal : } \\
1 \mathrm{~m}^{2} / \mathrm{ha}\end{array}$ & $\begin{array}{l}\text { - intensité maximale des } \\
\text { éclaircies : } 40 \% \\
\text { - rotation minimale : } 5 \text { ans } \\
\text { - prélèvement } \\
\text { minimal : } 1 \mathrm{~m}^{2} / \mathrm{ha} \\
\text { - nature minimale des } \\
\text { éclaircies : } 65 \% \\
\text { - facteur } \\
\text { maximal d'élancement : }\end{array}$ \\
\hline $\begin{array}{l}\left(H_{o}: \text { hauteur dominante }\right. \\
D_{g}: \text { diamètre moyen quadratique) }\end{array}$ & & $\frac{H_{o}}{D_{g}}=105-0,5^{*} H_{o}$ \\
\hline
\end{tabular}

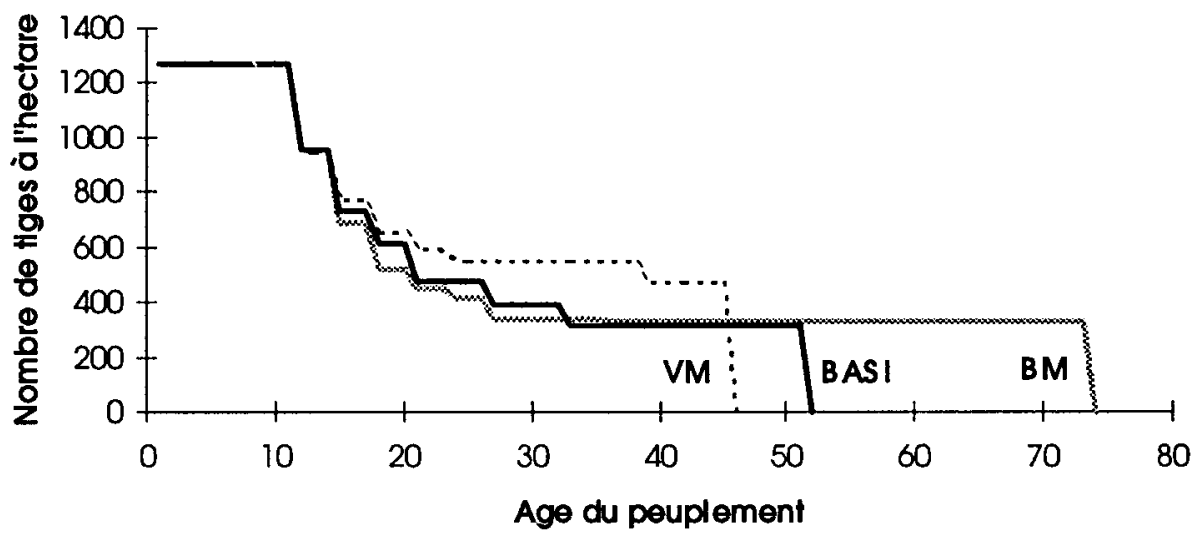

Figure 6. Évolution du nombre de tiges avec l'âge selon les critères $V M, B M, B A S I$ (3\%) pour le pin maritime. 
Tableau III. Résultats sylvicoles optimisés selon les critères $V M, B M, B A S I(3 \%)$ pour le pin maritime.

\begin{tabular}{|c|c|c|c|}
\hline $\begin{array}{l}\text { Caractéristiques } \\
\text { Valeur des critères : }\end{array}$ & $\begin{array}{c}\text { Max VM } \\
\mathbf{1 8 , 1} \mathbf{~ m}^{\mathbf{3}} / \mathbf{h a} / \mathbf{a n} \\
38521 \mathrm{~F} / \mathrm{ha} \\
3101 \mathrm{~F} / \mathrm{ha} / \mathrm{an}\end{array}$ & $\begin{array}{c}\text { Max BASI } \\
17,3 \mathrm{~m}^{3} / \mathrm{ha} / \mathrm{an} \\
\mathbf{4 8 7 2 5} \mathrm{F} / \mathrm{ha} \\
4075 \mathrm{~F} / \mathrm{ha} / \mathrm{an}\end{array}$ & $\begin{array}{c}\text { Max BM } \\
16,0 \mathrm{~m}^{3} / \mathrm{ha} / \mathrm{an} \\
36034 \mathrm{~F} / \mathrm{ha} \\
\mathbf{4 8 1 6 ~ F / h a / a n}\end{array}$ \\
\hline $1^{\text {re éclaircie année }}$ & 11 ans & 11 ans & 11 ans \\
\hline intensité & $26,9 \%$ & $24,7 \%$ & $24,7 \%$ \\
\hline $2^{e}$ éclaircie année & 14 ans & 14 ans & 14 ans \\
\hline intensité & $17,5 \%$ & $23,4 \%$ & $28,1 \%$ \\
\hline $3^{\mathrm{e}}$ éclaircie année & 17 ans & 17 ans & 17 ans \\
\hline intensité & $15,4 \%$ & $16,3 \%$ & $23,9 \%$ \\
\hline $4^{\mathrm{e}}$ éclaircie année & 20 ans & 20 ans & 20 ans \\
\hline intensité & $9,1 \%$ & $22,0 \%$ & $14,3 \%$ \\
\hline $5^{\mathrm{e}}$ éclaircie année & 23 ans & 26 ans & 23 ans \\
\hline intensité & $7,5 \%$ & $18,8 \%$ & $6,7 \%$ \\
\hline $6^{\mathrm{e}}$ éclaircie année & 38 ans & 32 ans & 26 ans \\
\hline intensité & $13,5 \%$ & $19,2 \%$ & $17,9 \%$ \\
\hline $7^{\text {e }}$ éclaircie année & & & 35 ans \\
\hline intensité & & & $4,4 \%$ \\
\hline Coupe finale année & 45 ans & 51 ans & 73 ans \\
\hline Nombre de tiges finales & 480 tiges & 315 tiges & 330 tiges \\
\hline Circonférence q. moyenne finale & $131 \mathrm{~cm}$ & $157 \mathrm{~cm}$ & $172 \mathrm{~cm}$ \\
\hline Diamètre q. moyen final & $42 \mathrm{~cm}$ & $50 \mathrm{~cm}$ & $55 \mathrm{~cm}$ \\
\hline
\end{tabular}

Tableau IV. Résultats sylvicoles optimisés selon les critères $V M, B M, B A S I(3 \%)$ pour l'épicéa commun.

\begin{tabular}{|c|c|c|c|}
\hline $\begin{array}{l}\text { Caractéristiques } \\
\text { Valeur des critères }\end{array}$ & $\begin{array}{c}\text { Max VM } \\
12,7 \mathbf{~ m}^{3} / \mathrm{ha} / \mathrm{an} \\
4907 \mathrm{~F} / \mathrm{ha} \\
1175 \mathrm{~F} / \mathrm{ha} / \mathrm{an}\end{array}$ & $\begin{array}{c}\text { Max BASI } \\
12,4 \mathrm{~m}^{3} / \mathrm{ha} / \mathrm{an} \\
11754 \mathrm{~F} / \mathrm{ha} \\
1926 \mathrm{~F} / \mathrm{ha} / \mathrm{an}\end{array}$ & $\begin{array}{c}\text { Max BM } \\
11,7 \mathrm{~m}^{3} / \mathrm{ha} / \mathrm{an} \\
10950 \mathrm{~F} / \mathrm{ha} \\
2 \mathbf{1 3 3} \mathbf{F} / \mathrm{ha} / \mathbf{a n}\end{array}$ \\
\hline $1^{\mathrm{re}}$ éclaircie année & 20 ans & 20 ans & 20 ans \\
\hline intensité & $38,3 \%$ & $40,0 \%$ & $40,0 \%$ \\
\hline $2^{\mathrm{e}}$ éclaircie année & 26 ans & 25 ans & 25 ans \\
\hline intensité & $20,3 \%$ & $38,9 \%$ & $38,9 \%$ \\
\hline $3^{e}$ éclaircie année & 33 ans & 30 ans & 30 ans \\
\hline intensité & $27,1 \%$ & $20,5 \%$ & $36,4 \%$ \\
\hline $4^{\mathrm{e}}$ éclaircie année & 38 ans & 44 ans & 41 ans \\
\hline intensité & $25,6 \%$ & $22,9 \%$ & $14,3 \%$ \\
\hline $5^{\text {e }}$ éclaircie année & 43 ans & 49 ans & 54 ans \\
\hline intensité & $12,5 \%$ & $18,5 \%$ & $16,7 \%$ \\
\hline $6^{e}$ éclaircie année & & 54 ans & 59 ans \\
\hline intensité & & $22,7 \%$ & $30,0 \%$ \\
\hline Coupe finale année & 46 ans & 59 ans & 69 ans \\
\hline Nombre de tiges finales & 420 tiges & 255 tiges & 210 tiges \\
\hline Circonférence q. moyenne finale & $97 \mathrm{~cm}$ & $122 \mathrm{~cm}$ & $135 \mathrm{~cm}$ \\
\hline Diamètre q. moyen final & $31 \mathrm{~cm}$ & $39 \mathrm{~cm}$ & $43 \mathrm{~cm}$ \\
\hline
\end{tabular}




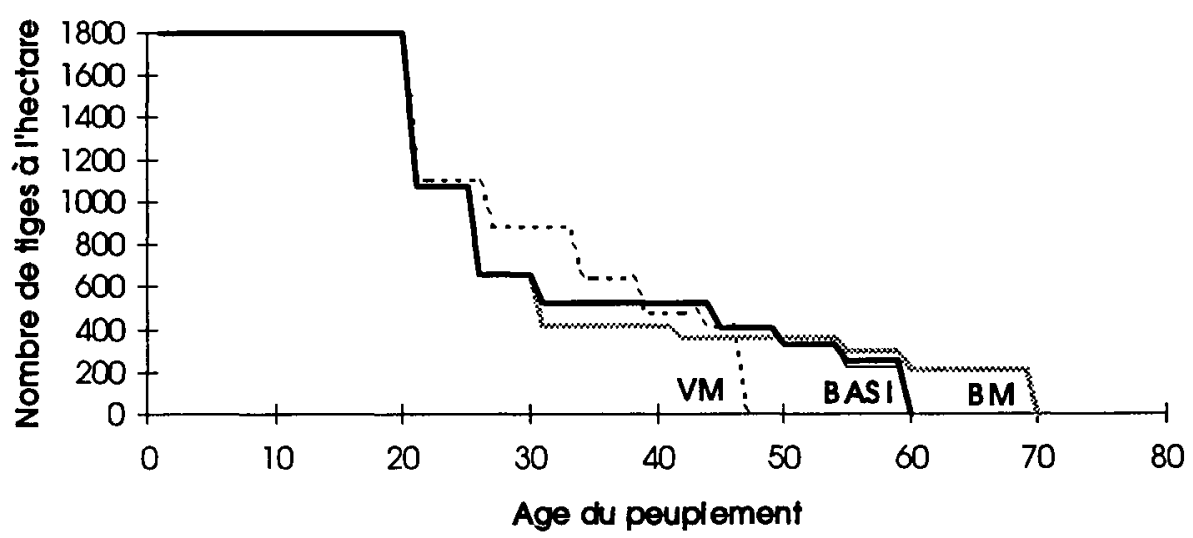

Figure 7. Évolution du nombre de tiges avec l'âge selon les critères $V M, B M, B A S I$ ( $3 \%$ ) pour l'épicéa commun.

sylvicole et non seulement de l'âge optimal d'exploitation.

Ils montrent tout d'abord que les âges optimaux d'exploitation se présentent bien dans l'ordre attendu, tel que le laissait prévoir le cas simplifié : la production moyenne en volume, et le bénéfice actualisé culminent avant le bénéfice moyen.

Dans ces exemples avec recettes et dépenses intermédiaires, le choix du critère joue non seulement sur l'âge optimal d'exploitation, mais encore sur le calendrier et l'intensité des éclaircies. De ce dernier point de vue, il s'avère que la sylviculture selon le critère physique $V M$ est toujours la moins dynamique. En ce qui concerne les éclaircies, la sylviculture maximisant le bénéfice moyen annuel $B M$ apparaît d'abord intensive, mais son dynamisme se réduit ensuite considérablement. À l'inverse, le critère de rentabilité induit une sylviculture plus soutenue et régulière, mais évidemment sur une durée d'autant plus courte que le taux est élevé.

La valeur du critère passe par un optimum dont la forme peut être étudiée en comparant les valeurs obtenues pour chaque critère aux trois âges d'exploita- bilité correspondant respectivement aux trois critères (voir les tableaux $I I I$ et $I V$ ). Il s'avère que le volume moyen annuel récolté $V M$ est le moins sensible à la variation de l'âge d'exploitation; il varie de $12 \%$ au maximum dans les cas étudiés, en étant un peu plus fort pour le pin maritime que pour l'épicéa commun. En revanche, le bénéfice moyen annuel et le bénéfice actualisé varient jusqu'à $45 \%$ pour le premier dans le cas du pin maritime et $58 \%$ pour le second dans le cas de l'épicéa commun.

Les critères analysés apparaissent en définitive adaptés à une stratégie d'optimisation aussi bien dans les exemples les plus proches de la réalité que dans le cas simplifié.

\section{RECHERCHE DE RENDEMENTS SOUTENUS}

Les critères de gestion qui ont été étudiés jusque là l'ont été dans le cadre d'un peuplement équienne donné. En réalité, on a rarement affaire à des peuplements isolés, mais plutôt à des forêts constituées 
de plusieurs peuplements d'âges différents, voire de peuplements inéquiennes ou jardinés. L'objectif à long terme consiste assez fréquemment, au moins pour certaines catégories de propriétaires, à rechercher un état d'équilibre qui puisse notamment se perpétuer dans le temps. Il s'agit ici de caractériser un état objectif possible à long terme.

Une telle démarche concerne aussi bien une futaie régulière, composée de peuplements équiennes, que des peuplements inéquiennes ou jardinés. D'une certaine façon, la structure inéquienne peut être vue comme une limite, quand la surface des peuplements tend à s'annuler, d'une structure régulière.

La description de l'état d'équilibre nécessite d'utiliser de nouvelles notations.

$D$ : dépenses annuelles à l'équilibre par unité de surface ;

$V:$ volume exploité annuellement à l'équilibre par unité de surface ;

$R$ : recettes annuelles à l'équilibre par unité de surface ;

$E$ : indice qualifiant les critères à l'équilibre à long terme.

L'état objectif est défini par un équilibre des classes d'âge ou une gradation des classes de diamètre qui puisse être maintenue stationnaire au cours du temps, notamment grâce aux interventions sylvicoles. Cette stationnarité ou invariance peut être requise à diverses échelles de temps. Elle est considérée ici à son niveau le plus fin : l'année. La forêt équilibrée produit ainsi annuellement les mêmes volume $V$, revenu $R$ et bénéfice $R-D$.

Dans le cas le plus général où elle est appliquée au bénéfice pour une forêt équilibrée, l'accumulation par unité de surface et sur une durée $n$ s'écrit :

$$
B T_{E}=n \cdot(R-D)
$$

Cette formule peut être retrouvée sans difficultés à partir de la formule (22) dans le cas d'une futaie régulière composée de $n$ peuplements équiennes d'âge gradué.

La productivité annuelle d'une forêt équilibrée se déduit de la production totale en raisonnant à l'année et non plus sur une période de $n$ années. On obtient l'expression suivante qui correspond à l'équation (23) :

$$
B M_{E}=R-D
$$

L'utilisation de critères de rentabilité sur une seule révolution peut être adaptée au cas d'une forêt équilibrée en remplaçant l'idée de révolution par celle de période annuelle :

$$
B A S F_{E}=\frac{R-D-r \cdot\left(F+S_{E}\right)}{1+r}
$$

où $S_{E}$ représente la valeur d'avenir, par unité de surface, de l'ensemble des peuplements.

Le critère précédent est logiquement annulé par le taux interne de rentabilité :

$$
T I R F_{E}=\frac{R-D}{F+S_{E}}
$$

Cette formule illustre bien le fait que la rentabilité est une mesure des profits réalisés relativement aux capitaux investis. Elle suggère aussi que l'équivalent du taux $T I R$ ignorant les immobilisations (fonds et peuplement) n'est pas définie dans ce cas.

Enfin, le bénéfice actualisé à l'infini correspond à la valeur d'avenir calculée de l'ensemble de la forêt, fonds et peuplements, en tant que somme des revenus nets futurs actualisés. Comme la forêt engendre chaque année un revenu net égal à $R-D$, il s'écrit :

$$
B A S I_{E}=\frac{R-D}{r}
$$




\subsection{Quelques commentaires sur ces critères relatifs à l'état objectif à long terme}

De nombreuses discussions ont porté sur celui des critères qui s'avérait le plus conforme à la théorie économique dans le cas d'une forêt équilibrée considérée à long terme. Faustmann [12-14] déclare nettement que sa théorie est valable aussi bien pour un peuplement considéré isolément que pour une forêt. Cependant, Duerr [10] publie un critère dont l'ambition est de prendre en compte trois coûts cumulés qui sont subis lorsque s'allonge la révolution :

- l'attente de l'exploitabilité (intégrée dans un bénéfice actualisé simple) ;

- le coût du report dans le temps de toutes les révolutions ultérieures (intégré dans le $B A S I)$;

- le coût de l'équilibre, qui correspond à l'immobilisation permanente d'un capital sur pied, et qui constitue pour Duerr, la principale innovation de son critère.

Ce critère reste peu utilisé, à l'exception de quelques auteurs qui s'y réfèrent tels que Nautiyal [32] ou Buongiorno [3, 4]. Oderwald et Duerr (1990) réaffirment alors l'intérêt de ce critère et suscitent de nombreuses critiques, notamment celle de Chang [5]. Ce dernier objecte que la valeur du peuplement prise en compte par Duerr pour déterminer le coût de l'immobilisation est la valeur de consommation du peuplement, c'est-à-dire la valeur d'une vente effective des arbres; il remarque qu'en utilisant la valeur d'avenir du même peuplement, qui anticipe la valeur des récoltes futures et dépasse la valeur de consommation tant que le peuplement n'a pas atteint l'âge d'exploitabilité, on retrouve le critère de Faustmann, c'est-à-dire la maximisation du BASI. Il n'a donc pas semblé nécessaire de présenter ici le critère de Duerr.
Par ailleurs, l'optimisation des critères à long terme fournit des renseignements intéressants mais ne conduit pas, en général, à la gestion optimale d'une forêt réelle dans la mesure où les conditions initiales influent largement sur cet optimum ; il faut alors envisager explicitement l'étape de la transition entre la situation actuelle de la forêt et un état objectif à long terme.

\section{GESTION DES PEUPLEMENTS EN COURS DE CROISSANCE}

Le raisonnement a porté jusqu'ici sur le long terme, considérant un peuplement depuis son origine 0 , jusqu'à son exploitation finale à l'âge $n$, ou bien encore dans le cadre d'une gestion équilibrée. Mais on gère beaucoup plus fréquemment des peuplements existants, qu'il importe de traiter du mieux possible à titre transitoire et de conduire à l'exploitation finale ou à l'équilibre. Il est alors nécessaire de définir la gestion convenant le mieux aux peuplements actuels avant qu'ils atteignent soit l'exploitabilité, soit l'équilibre.

Lorsqu'on se trouve en présence de peuplements équiennes en cours de croissance, leur âge n'est pas toujours très bien connu. Dans le cas d'un peuplement inéquienne, l'âge n'est pas une variable adéquate. C'est pourquoi le temps constitue ici une meilleure référence que l'âge. On notera donc :

$t \quad$ : temps, variant de $o$ à $u$;

$D(t)$ : dépenses annuelles par unité de surface au temps $t$;

$R(t)$ : revenus annuels encaissés par unité de surface au temps $t$

$C$ : indice rappelant qu'on s'intéresse à un peuplement ou à une forêt en cours de croissance. 
En ce qui concerne tout d'abord l'accumulation, elle peut être comptée sans difficultés à partir de l'instant présent :

$$
B T_{C}=\sum_{t=0}^{t=u}[R(t)-D(t)]
$$

En ce qui concerne ensuite les critères de productivité, il s'agit tout d'abord de se fixer un objectif à long terme, $B M^{*}$, dont la mise en ouvre pourra commencer dès après, respectivement, la récolte du peuplement actuel ou l'obtention de l'équilibre. L'analyse effectuée dans la première section (équation (6) et commentaires associés) conduit alors à affirmer qu'il convient de poursuivre la gestion actuelle sur une durée $u$ telle que la productivité moyenne sur cette période soit supérieure à la productivité de référence et de l'arrêter dès que la production courante égale cette dernière. La gestion optimale consiste alors à maximiser :

$$
B M_{C}=\left(\sum_{t=0}^{t=u}[R(t)-D(t)]\right)-u \cdot B M^{*}
$$

Ce critère n'a pas la même nature que celui qui a été défini à long terme dans le cas équienne par l'équation (33) mais, dans les conditions où ils peuvent être tous deux utilisés, ils conduisent à la même gestion optimale. L'expression (28) vient donc généraliser l'utilisation de la productivité comme base de gestion.

Lorsqu'on utilise un bénéfice actualisé sur une seule révolution, tel que $B A S F$, l'analyse peut être conduite sans difficultés en considérant seulement les recettes et dépenses futures jusqu'à l'exploitation du peuplement ou l'avènement de l'équilibre :

$$
\begin{aligned}
& \text { BASF }_{o}=-\left(F+S_{C}\right) \\
& +\left(\sum_{t=o}^{t=u} \frac{R(t)-D(t)}{(1+r)^{t}}\right)+\frac{F}{(1+r)^{u}}
\end{aligned}
$$

où $S_{C}$ représente la valeur d'avenir du peuplement ou des peuplements de la forêt au temps présent.
À ce bénéfice actualisé correspond le taux interne de rentabilité :

$T I R F_{C}$ racine de l'équation :

$$
\begin{aligned}
& F+S_{C}=\left(\sum_{t=\iota "}^{t=u} \frac{R(t)-D(t)}{\left(1+T I R F_{C}\right)^{t}}\right) \\
& +\frac{F}{\left(1+T I R F_{C}\right)^{u}}
\end{aligned}
$$

Dans son principe qui consiste à maximiser la somme actualisée des revenus nets futurs, le bénéfice actualisé à l'infini peut être appliqué directement à tout peuplement ou ensemble de peuplements en cours de croissance à condition que les revenus nets futurs correspondants puissent être anticipés. C'est même une de ses importantes propriétés que d'être cohérent sur le plan temporel : comparer, selon ce critère, deux gestions sylvicoles en considérant le peuplement ou les peuplements depuis un instant origine et après un délai donné fournit toujours le même résultat quel que soit ce délai. Il suffit donc d'utiliser l'adaptation suivante :

$$
B A S I_{C}=\left(\sum_{t=n}^{t=u} \frac{R(t)-D(t)}{(1+r)^{t}}\right)+\frac{B A S I^{*}}{(1+r)^{u}}
$$

où $B A S I^{*}$ représente la valeur actualisée de la séquence infinie de bénéfices qui commencera dans $u$ années après régénération du peuplement ou avènement de l'équilibre. Quant au premier terme, il constitue le cumul des bénéfices actualisés au cours de la période de transition de durée $u$.

Plusieurs auteurs ont notamment étudié le poids des conditions initiales sur la conduite vers un état d'équilibre $[22,28$, $32,39]$. Un cas particulier fréquemment considéré est celui où l'on reboise progressivement un sol nu pour constituer à terme une forêt équilibrée. 


\section{QUELQUES RÉFLEXIONS DANS LE CAS D'UN MARCHÉ FINANCIER IMPARFAIT}

L'ensemble des considérations précédentes est placé sous l'hypothèse d'un marché financier parfait : le taux d'intérêt applicable aux emprunts et aux placements est le même, et les montants que l'on peut emprunter et placer à ce taux ne sont pas limités. Or, si certains types d'instruments financiers essaient de se rapprocher de cette perfection (plans et comptes d'épargne logement notamment en ce qui concerne l'égalité des taux), les marchés financiers réels ne sont pas parfaits. L'organisme prêteur doit en effet couvrir le risque qu'il prend en devenant créancier, rembourser ses frais de gestion et dégager un profit. Ces trois éléments sont financés par la différence entre taux de prêt et taux de placement.

Quelles conséquences pour le consommateur découlent de la nature du marché financier? Soit un consommateur qui connaisse ses revenus présents et anticipe ses revenus futurs. Pour simplifier et pouvoir réaliser une représentation graphique, raisonnons sur ces seules deux périodes de temps. S'il consomme exactement ses revenus à chaque période, il se situe par exemple au point $\mathrm{A}_{0}$ des figures 8.a et 8.b. S'il a la possibilité d'épargner ou d'emprunter de l'argent, il pourra au contraire s'éloigner de ce point dans un sens ou dans l'autre.

Dans le cas d'un marché financier parfait (figure 8.a), l'ensemble des possibilités de consommation épuisant le revenu est constitué par la droite de budget $\mathrm{A}_{1} \mathrm{~A}_{2}$. Compte tenu de ses préférences entre le présent et le futur, le consommateur trouvera son équilibre au point $A^{*}$ où son taux de préférence intertemporel (pente de la tangente à la courbe d'indifférence) est égal au taux du marché financier (pente de la droite de budget). Dans le cas d'un marché financier imparfait (figure 8.b), a)

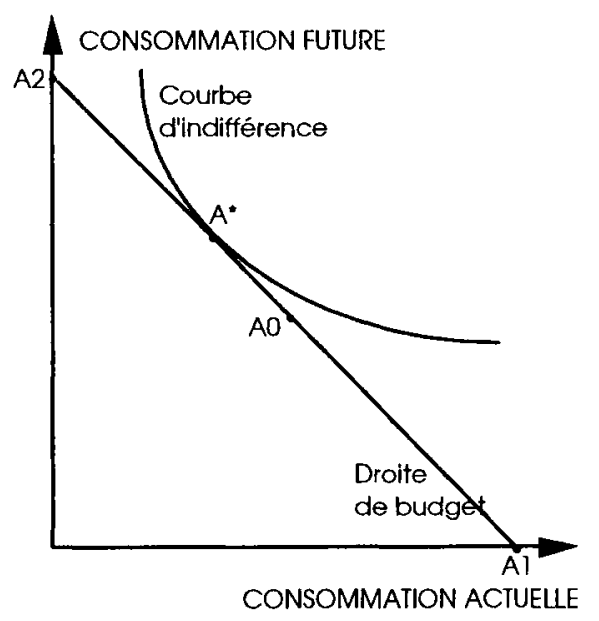

b)

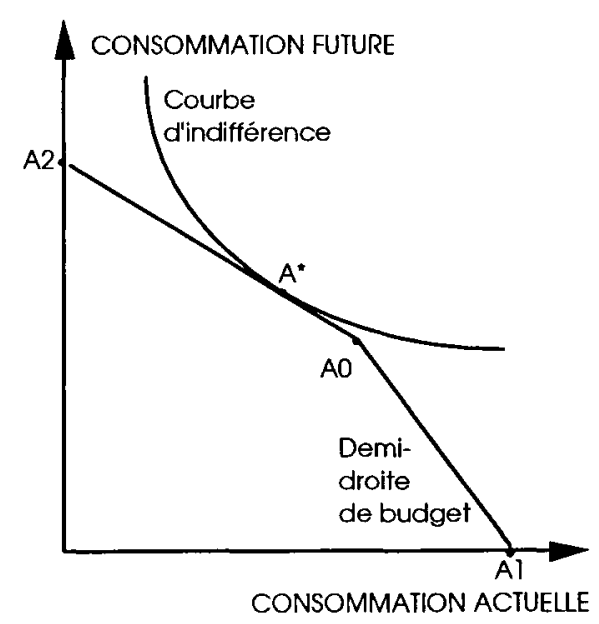

Figure 8. Comparaison entre l'équilibre du consommateur sur un marché financier parfait (a) et imparfait (b) dans le cas simplifié de deux périodes de temps. 
les possibilités qui épuisent les ressources du consommateur sont celles des deux demi-droites $\mathrm{A}_{0} \mathrm{~A}_{1}$ et $\mathrm{A}_{0} \mathrm{~A}_{2}$ dont les pentes sont respectivement données par le taux d'emprunt $\left(r_{\max }\right)$ et le taux de placement $\left(r_{\min }\right.$, plus faible). L'équilibre du consommateur a ainsi de fortes chances de se trouver en $A_{0}$, point singulier, mais il peut aussi s'en écarter, comme c'est le cas sur la figure.

Des analyses ont été conduites dans le cas d'un marché financier imparfait, par exemple par Frayssé, Moreaux \& Terreaux [15], par Terreaux [44] et par Morel \& Terreaux [30]. Elles consistent à distinguer deux situations. La première correspond à des revenus présents plus forts que la consommation (cas de la figure $8 . b$ ) : le propriétaire épargne au taux $r_{\text {min }}$; $s$ 'il décide d'investir en forêt, il se privera d'une rémunération financière sur la base de ce taux qu'il va donc choisir comme taux d'actualisation pour évaluer son projet. La seconde situation est inverse : le propriétaire est emprunteur sur le marché financier au taux $r_{\max }$; s'il décide d'investir en forêt, il devra emprunter une somme supplémentaire et subira des frais financiers selon ce taux qu'il prendra donc comme taux d'actualisation. Finalement, le taux d'actualisation choisi dépend de la situation financière du propriétaire.

De manière pratique, les calculs d'actualisation peuvent être faits en utilisant le taux $r_{\min }$ lorsque le bilan financier prévisionnel de l'année est positif, et le taux $r_{\text {max }}$ dans le cas contraire. Les critères de gestion doivent alors être pris à l'échelle de l'ensemble de la forêt, voire du patrimoine total du propriétaire. D'où l'importance, non seulement sur le plan technique mais aussi d'un point de vue économique, de la réalisation d'un plan de gestion.

En poussant plus loin le raisonnement, on comprend intuitivement et démontre rigoureusement [30] qu'un propriétaire trouve un intérêt dans une situation proche de l'équilibre, ou au moins qui lui permette constamment de dégager des bénéfices, donc d'éviter un emprunt, et finalement d'actualiser ses dépenses et recettes à un taux relativement faible, rendant les investissements plus attractifs.

De la même façon, la valeur d'une forêt est d'autant plus importante que le taux d'actualisation est faible, d'abord parce que le fonds se trouve renchéri, ensuite parce que la perte d'avenir est augmentée. La forêt représente donc une valeur plus grande pour les épargnants que pour les emprunteurs. On comprend également que les investissements aient une plus grande probabilité de voir le jour, d'être rentables, lorsqu'ils peuvent être autofinancés.

La prise en compte de l'imperfection des marchés est possible, mais nécessite une approche d'ensemble du patrimoine des propriétaires forestiers. Elle est par conséquent relativement lourde à mettre en œuvre, et doit être adaptée à chaque cas particulier. C'est pourquoi il n'est pas opportun de formuler autrement les critères correspondants sinon en faisant remarquer que, dans le cas de marchés imparfaits, les bénéfices actualisés doivent être adaptés de la manière suivante :

$\begin{cases}r(t)=r_{\min } & \text { les années bénéficiaires } \\ r(t)=r_{\max } & \text { les années déficitaires }\end{cases}$

\section{CONCLUSIONS}

Cet article a essayé de présenter de manière aussi exhaustive et claire que possible l'ensemble des critères susceptibles d'être utilisés dans une approche économique de la gestion forestière. Les hypothèses et conditions d'utilisation propres à chacun d'eux ont été précisées. Contribuant ainsi à créer un écart entre situations réelles et théoriques, elles expliquent la multiplicité des critères dont aucun ne peut avoir la prétention d'être toujours 
préféré aux autres. Mais elles permettent également de donner aux chercheurs et aux gestionnaires toutes les informations nécessaires sur le choix de l'un d'entre ces critères. Cet aspect pédagogique s'avère largement indispensable dans la mesure où des critères mis en exergue voilà plus d'un siècle semblent faire l'objet d'applications peu nombreuses. C'est pourquoi il a semblé bon d'insister également sur des points historiques.

La présentation qui est traditionnellement réalisée s'appuie sur la formulation simplifiée traitée dans le cas d'une gestion à long terme pour les peuplements équiennes. Elle a été complétée ici par la considération d'une forêt équilibrée, par celle du court terme, et par le passage du cas simplifié au cas réel, tant en ce qui concerne les aspects techniques que financiers. Cette analyse étendue montre ainsi une bonne convergence entre les résultats fournis par la théorie simplifiée et des applications plus complètes. Elle montre également, d'une certaine façon, l'adaptabilité de la théorie de base à des situations plus générales.

Enfin, il convient de garder à l'esprit que les critères qui ont été analysés reposent essentiellement sur des bases marchandes dans des conditions déterministes, et devraient être complétés par la considération de biens et services non marchands et l'introduction de l'incertitude et des risques qui pèsent sur la gestion forestière.

\section{REMERCIEMENTS}

Les recherches qui ont conduit à cet article ont été financées par l'Union Européenne dans le cadre du programme AIR (Forest planning and management tools), par la direction générale de l'enseignement et de la recherche du ministère de l'Agriculture et de la Pêche dans le cadre de la formation par la recherche, et par la région Aquitaine sous la forme d'une demi bourse de thèse.

\section{RÉFÉRENCES}

(1) Baudrillart J.J., Traité général des eaux et forêts, chasses et pêches. ; dictionnaire général raisonné et historique des Eaux et Forêts, Arthus Bertrand et Mme Huzard, Paris. Tome 1,816 p.,tome $2: 1006 \mathrm{p}, 1825$.

[2] Brard Y, Domaines public et privé des personnes publiques. Dalloz, connaissance du droit, Paris, 117 p, 1994.

[3] Buongiorno J., Dahir S., Lu H.C., Lin C.R., Tree size diversity and economic returns in uneven-aged forest stands. For. Sci. 40 (1) (1994) 83-103.

[4] Buongiorno J., Peyron J.L., Houllier F., Bruciamacchie M., Growth and management of mixed-species, uneven-aged forests in the French Jura : implications for economic returns and tree diversity. For. Sci. 41 (3) (1995) 397-429.

[5] Chang S.J., Comment II on Oderwald and Duerr, For. Sci. 361 (1990) 177-179.

[6] Chapman H.H., Forest management. J.B Lyon Company Publishers, Albany, NY. États-Unis, 1931.

[7] Cotta H., 1819 a. Entwurf einer anweisung zur Waldwerthberechnung (Tharand, décembre 1817 à la préface). Dresde, 136 p.

[8] Cotta H., 1819 b. Entwurf einer anweisung zur Waldwerthberechnung. Traduction et conversion en mesures de France In : Salomon D., Traité de l'aménagement des forêts, enseigné à l'école royale forestière ; tome second, Bureau de l'Almanach du Commerce. Paris, Thinus et Baret, Mulhouse \& Grimblot, Nancy, pp. 219-372, 1837.

19] Dehem R., Histoire de la pensée économique des mercantilistes à Keynes. Dunod Paris et les Presses de l'Université Laval, Québec. 448 p, 1984.

[10] Duerr W.A., Fundamentals of forestry economics. McGraw-Hill Book Company, New York, Toronto, London, 579 p., 1960.

[11] Duhamel du Monceau H.L., Traité complet des bois et des forêts; seconde partie de l'exploitation des bois, ou moyens de tirer un parti avantageux des taillis, demi-futaies et hautes-futaies, et d'en faire une juste estimation avec la description des arts qui se pratiquent dans les forêts. Paris : H.L. Guérin et L.F. Delatour, $1^{\text {re }}$ partie : 430 p. + planches ; $2^{\mathrm{c}}$ partie : $708 \mathrm{p}, 1964$

[12] Faustmann M., Auflösung einer Aufgabe der Waldwerthberechnung, Allgemeine Forstund Jagdzeitung, Frankfurt a. M., pp. 285-295, 1849a.

[13] Faustmann M., Berechnung des Wertes, we]chen Waldboden, sowie noch nicht haubare Holzbestände für die Waldwirtschaft besit- 
zen. Allgemeine Forst- und Jagdzeitung, Frankfurt a. M., pp. 441-455, 1849 b.

[14] Faustmann M., Calculation of the value which forest land and immature stands possess for forestry. Translated by Linnard, In : Martin Faustmann and the evolution of discounted cash flow. Commonwealth Forestry Institute. University of Oxford, 1968, pp. 27-55, 1849c.

[15] Frayssé J., Moreaux M., Terrcaux J. Ph., Actualisation et gestion forestière. Cahiers d'Économie et Sociologie Rurales, n ${ }^{\circ}$ 15-16, pp. 111-125, 1990 .

[16] Gane M., Introduction. In : Martin Faustmann and the evolution of discounted cash flow. Commonw. For. Institute, University of Oxford, pp. 5-16, 1968.

[17] Guillon, P., La rentabilité des reboiscments, étude méthodologique. Inra, Nancy, 93 p, 1977.

[18] Guo B., Recherche d'une sylviculture optimale à long terme pour les peuplements forestiers équiennes; formulation, résolution, applications. Thèse de doctorat Engref, Paris, Nancy 242 p, 1994.

[19] Hartig G.L., 1796. Anweisung zur Holtzzucht für Förster. Marburg. |Traduit en françàis par Baudrillart, 1805, sous le titre : Instruction sur la culture des bois à l'usage des forestiers. Paris. 172 p.l

[20] Hiley W.E., The economics of forestry. Clarendon Press, Oxford, 256 p, 1930.

[21] Houllier F., Leban J.M., Colin F.. Modelling growth of Norway spruce for timber quality assessment, For. Ecol. Manage. 74 (1995) 91-102.

[22] Hultkranz L., A note on the optimal rotation period in a synchronized normal forest. For. Sci., 37 (4) 1991, 1201-1206.

[23] Johansson P.O., Löfgren K.G., The economics of forestry and natural resources. Basil Backwell Lid, New York, 292 p. 1985.

[24] Johnston D.R., Grayson A.J., Bradley R.T., Forest planning. Faber and Faber Lid, London, $541 \mathrm{p}, 1967$.

[25] König G., Die Forstwirtschaft für den Forstdienst bearbeitet \& dic Forst-Mathematik mit Anweisung zur Forstvermessung. Holtzschätzung und Waldwethberechnung, nebst Hülfstafeln für Forstschätzer. Gothat. 128 p, 1835.

[26] Le Duc P., Euvres agronomiques el forestières de Varenne de Fenille ; études précédées d'une notice bibliographique. J. Rothschild, Paris.512 p. 1869.

[27] Lemoine B., Croissance et production du pin maritime. Recherche d'un modèle ct d'une méthode. Ann. Sci. For., 39 (4) (1991) 321-354.
[28] Lohmander P., The rotation age, the constrained Faustmann problem and the initial conditions. Scandinavian Forest Economics, $\mathrm{n}^{\circ} 29-1987$, proceedings of the biennal meeting, pp. 101-129, 1987.

[29] Mantel K., Pacher J., Forstliche Biographie vom 14. Jahrhundert bis zur Gegenwart, zugleich eine Einführung in die forstliche Literaturgeschichte; Band 1. Verlag M. \& H. Schaper, Hannover, 441 p., 1976.

[30] Morel M. \& Terreaux J. Ph., L'estimation de la valeur des forêts à travers un exemple : entre simplification abusive et complexité du réel. Revue Forestière Française (à paraître), 1995.

[31] Nanquette H., Cours d'aménagement des forêts, enseigné à l'école impériale forestrière. Bouchard-Huzard, Paris \& Grimblot, Nancy, 327 p, 1860 .

[32] Nautiyal J.C., 1983. Toward a method of uneven-age forest management based on the theory of financial maturity. For. Sci. 29 (1), 47-59.

133] Nepveu G., La variabilité du bois. In : Jodin P., Le matériau bois; seconde édition mise en forme, Arbolor, Nancy, 56 p, 1991.

134) Newman D.H., The optimal forest rotation : a discussion and annoted bibliography. USDA-Forest Service, general technical report SE-48, Asheville, NC, U.S., 47p, 1988.

[35] Oderwald R.G., Duerr W.A., König-Faustmannism : a critique. For. Sci., 36 (1) (1990) 169-174.

136] O.N.F., Manucl d’aménagement. 20 édition rédigée par L. Bourgenot. Office National des Forêts, Paris, 202 p, 1969

[37] O.N.F., Manuel d’aménagement. $3^{\circ}$ édition rédigéc par J. Dubourdieu. Office National des Forêts, Paris, 151 p, 1989.

[38] O.N.F., Instruction sur l'aménagement forestier pour les forêts relevant du régime forestier. Instruction rédigée sous la responsabilité de J. Dubourdicu. Office National des Forêts, Paris, 97 p, 1994.

139] Parades G.L., Brodie J.D., Land value and the linkage between stand and forest level analyses. Land economics, vol. 65, n'2, may 1989, pp. 158-166, 1989.

[40] Pardé 1 ... Traité pratique d'aménagement des forĉts. P.U.F., Paris, 547 p, 1930.

141] Samuelson P.A., Economics of forestry in an evolving society. Economic Inquiry, vol. XIV, déc., pp. 466-492 (texte présenté initialement au Symposium « The Economics of Sustained Yield Forestry », organisé par l'université de létat de Washington, à Scattle, le 23 nov. 1974), 1976.

1421 Schaeffer L., Principes d'estimation forestière, $2^{\mathrm{c}}$ édition. Enef, Nancy, 184 p, 1960. 
[43] Speidel G., Forstliche Betriebswirtschaftslehre. Verlag Paul Parey, Hamburg \& Berlin, 292 p, 1967.

[44] Terreaux J. Ph., Critères d'investissements en forêt. Rapport technique Gremacq 8906 , miméo, Université de Toulouse I, 1989.

[45] Terreaux J. Ph., Principe de gestion des investissements en forêt. Thèse de doctorat, Université de Toulouse I. 374 p, 1990.

[46] Vannière B., Cours d'aménagement ; $2^{\mathrm{c}}$ partie : aménagement général. Engref, Nancy, $81 \mathrm{p}, 1981$.

[47] Varenne de Fenille P., Observations sur l'aménagement des forêts, et particulièrement des forêts nationales (présentées à l'Assemblée Nationale par la Société Royale d'Agriculture, le 9 Juin 1791). Société Royale d'Agriculture, Paris. 88p, 1791.

[48] Von Gehren., Ueber Geldwerthbestimmung des holzleeren Waldbodens. Allgemeine Forst- und Jagdzeitung, Frankfurt a. M, 1849 a.

[49] Von Gehren., On the determination of the money value of bare forest land. Translated by Linnard, In : Martin Faustmann and the evolution of discounted cash flow. Commonwealth Foresrty Institute, University of Oxford, 1968, pp. 19-26, 1849 b. 